\title{
GDF15 induces immunosuppression via CD48 on regulatory $T$ cells in hepatocellular carcinoma
}

\author{
Zhaowei Wang, ${ }^{1}$ Lei He, ${ }^{1}$ Weina Li, ${ }^{1}$ Chuanyang Xu, ${ }^{1}$ Jieyu Zhang, ${ }^{1}$ \\ Desheng Wang, ${ }^{2}$ Kefeng Dou, ${ }^{2}$ Ran Zhuang, ${ }^{3}$ Boquan Jin, ${ }^{3}$ Wei Zhang, ${ }^{1}$ \\ Qiang Hao, ${ }^{1}$ Kuo Zhang, ${ }^{1}$ Wangqian Zhang, ${ }_{1}^{1}$ Shuning Wang, ${ }^{1}$ Yuan Gao, ${ }^{1}$ \\ Jintao Gu, ${ }^{1}$ Lei Shang, ${ }^{4}$ Zhijun Tan, ${ }^{4}$ Haichuan Su, ${ }^{5}$ Yingqi Zhang, ${ }^{1}$ Cun Zhang, ${ }^{1}$ \\ Meng Li (D) ${ }^{1}$
}

To cite: Wang Z, He L, Li W, et al. GDF15 induces immunosuppression via CD48 on regulatory $T$ cells in hepatocellular carcinoma. Journal for ImmunoTherapy of Cancer 2021;9:e002787. doi:10.1136/jitc-2021-002787

- Additional supplemental material is published online only. To view, please visit the journal online (http://dx.doi.org/10. 1136/jitc-2021-002787).

ZW, LH and WL contributed equally.

Accepted 22 July 2021
Check for updates

(c) Author(s) (or their employer(s)) 2021. Re-use permitted under CC BY-NC. No commercial re-use. See rights and permissions. Published by BMJ.

For numbered affiliations see end of article.

\section{Correspondence to \\ Dr Meng Li; \\ limeng@fmmu.edu.cn}

Dr Cun Zhang;

zhangcun@fmmu.edu.cn

Dr Yingqi Zhang;

zhangygh@fmmu.edu.cn

\section{ABSTRACT}

Background $A$ better understanding of the molecular mechanisms that manifest in the immunosuppressive tumor microenvironment (TME) is crucial for developing more efficacious immunotherapies for hepatocellular carcinoma (HCC), which has a poor response to current immunotherapies. Regulatory T (Treg) cells are key mediators of HCC-associated immunosuppression. We investigated the selective mechanism exploited by HCC that lead to Treg cells expansion and to find more efficacious immunotherapies.

Methods We used matched tumor tissues and blood samples from 150 patients with HCC to identify key factors of Treg cells expansion. We used mass cytometry (CyTOF) and orthotopic cancer mouse models to analyze overall immunological changes after growth differentiation factor 15 (GDF15) gene ablation in HCC. We used flow cytometry, coimmunoprecipitation, RNA sequencing, mass spectrum, chromatin immunoprecipitation and Gdf15 ${ }^{-1}$, OT-I and GFP transgenic mice to demonstrate the effects of GDF15 on Treg cells and related molecular mechanism. We used hybridoma technology to generate monoclonal antibody to block GDF15 and evaluate its effects on HCC-associated immunosuppression.

Results GDF15 is positively associated with the elevation of Treg cell frequencies in patients wih HCC. Gene ablation of GDF15 in HCC can convert an immunosuppressive TME to an inflammatory state. GDF15 promotes the generation of peripherally derived inducible Treg (iTreg) cells and enhances the suppressive function of natural Treg (nTreg) cells by interacting with a previously unrecognized receptor CD48 on T cells and thus downregulates STUB1, an E3 ligase that mediates forkhead box P3 (FOXP3) protein degradation. GDF15 neutralizing antibody effectively eradicates HCC and augments the antitumor immunity in mouse.

Conclusions Our results reveal the generation and function enhancement of Treg cells induced by GDF15 is a new mechanism for HCC-related immunosuppression. CD48 is the first discovered receptor of GDF15 in the immune system which provide the possibility to solve the molecular mechanism of the immunomodulatory function of GDF15. The therapeutic GDF15 blockade achieves HCC clearance without obvious adverse events.

\section{INTRODUCTION}

Cancer immunotherapies have led to remarkable clinical responses in a broad spectrum of advanced cancers, but their efficacies are not uniform among cancer types. ${ }^{1}$ For instance, the objective response rate to nivolumab (anti-programmed cell death protein 1 (antiPD-1) antibody) in patients with advanced hepatocellular carcinoma (HCC) is $14 \%,{ }^{2}$ which is inferior to that in patients with advanced melanoma $(31 \%-44 \%),{ }^{3}$ renal cell carcinoma $(22 \%-25 \%)^{4}$ and other cancer types. ${ }^{5}$ A better understanding of the molecular mechanisms that manifest in the immunosuppressive tumor microenvironment (TME) is crucial for developing more efficacious immunotherapies and combinations for HCC.

Regulatory $\mathrm{T}$ (Treg) cells are key mediators of tumor-associated immunosuppression, and a surge of studies in various types of tumors including HCC have highlighted the elevated numbers of Treg cells in the TME and circulation of patients with cancer. ${ }^{6-9}$ Recently, single-cell analysis of tumor-infiltrating lymphocytes (TILs) further confirmed the expansion of Treg cells in HCC and revealed their highly suppressive nature. ${ }^{9}$ In addition, Treg cell-mediated immune suppression plays important roles in both HCC-induced immune tolerance and the tolerogenic liver environment of chronic viral infection, a major cause and promoter of HCC. ${ }^{10}$ More importantly, the immunosuppressive characteristics of Treg cells in HCC are not as controversial as in other individual tumor types. ${ }^{112}$ Therefore, targeting Treg cells is an appealing approach to evoke an efficient antitumor immune response in HCC. However, in a wide variety of preclinical cancer models, punctual ablation of Treg cells results in rapid 
tumor regression and extensive immune cells alterations within the TME ${ }^{13}{ }^{14}$ but profound systemic autoimmune and inflammatory consequences ensue. ${ }^{15}$ A deep understanding of the selective mechanisms exploited by HCC that lead to the expansion of Treg cells in tumors and the circulation, especially those that are dispensable for maintaining crucial physiological functions and Treg cell development, will provide improved strategies for orchestrating the immune system to eradicate cancers without deleterious responses.

Growth differentiation factor 15 (GDF15) is a distant transforming growth factor $\beta$ (TGF- $\beta$ ) family member that is expressed at a very low level under physiological conditions but strongly upregulated in inflammationrelated diseases and cancers. ${ }^{16-18}$ Recently, the roles of GDF15 to induce cachexia, anorexia and loss of body weight were deeply elucidated. ${ }^{19-22}$ However, as a cytokine that can be induced by inflammation, its immunomodulatory function is controversial ${ }^{1723-26}$ and, especially, its effects on Treg cells remain largely unknown. Here, we identified that GDF15 upregulates in HCC and it is a key promoter of Treg cells, which promotes the generation of peripherally derived inducible Treg (pTreg) cells and modulates the suppressive function of natural Treg (nTreg) cells through a previously unrecognized receptor CD48 on $\mathrm{T}$ cells and post-transcriptional mechanism. This is a new molecular mechanism for immunomodulatory function of GDF15. More importantly, our results support that GDF15 targeting may achieve 'the idealized outcome of optimal immunotherapy': tumor clearance without obvious adverse events in HCC.

\section{MATERIALS AND METHODS}

The complete experimental protocols are described in the online supplemental material.

\section{RESULTS}

\section{GDF15 is positively associated with the elevation of Treg cell frequencies in HCC}

To identify the selective mechanisms exploited by tumors to upregulate the Treg cell frequency in patients with tumor, we sorted $\mathrm{CD} 3^{+} \mathrm{CD} 4^{+} \mathrm{T}$ cells from the tumor tissues of 60 treatment-naive patients with HCC into CD25 and CD25 $5^{\text {hi }}$ populations by gating on the top $4 \%$ of blood $\mathrm{CD}^{+} \mathrm{T}$ cells from volunteers according to a previously reported method. ${ }^{9}$ The expression of FOXP3, the master transcription factor of Treg cells, in the gated CD25 and $\mathrm{CD} 25^{\text {hi }}$ populations confirmed that the Treg cells were correctly enriched (online supplemental figure 1A). Then, from the above 60 patients, we selected 8 patients whose tumors had a relatively high Treg frequency and 8 patients whose tumors had a relatively low Treg frequency among $\mathrm{CD}^{+}$TILs and performed RNA sequencing (RNA-seq) on their tumor samples (figure 1A and online supplemental table 1). The selected patients with a relatively high Treg cell frequency among $\mathrm{CD} 4^{+}$TILs also had a relatively high Treg cell frequency in draining lymph nodes (figure 1B) and in peripheral blood (figure 1C). The cumulative distributions of the differentially expressed messenger RNAs (mRNAs) in these two types of tumors showed that Treg cell-related genes identified by gene ontology (GO) terms were upregulated in tumors with a relatively high Treg cell frequency (online supplemental figure 1B), which validated the relevance of our samples for sequencing. Intriguingly, among the differentially expressed mRNAs in these two types of tumors, we failed to find TGF- $\beta$, a canonical molecule required for the maintenance of pTreg cells in vivo and the generation of in vitro-induced Treg cells (iTreg cells), ${ }^{27} 28$ but instead discovered GDF15, a distant member of the TGF- $\beta$ superfamily (figure 1D). Overexpression of GDF15 in tumors with a relatively high Treg cell frequency was confirmed by western blot analysis, reverse transcription quantitative PCR (qRT-PCR) and immunofluorescence (IF) staining (online supplemental figure 1C-E).

Next, we analyzed another 90 patients with HCC to evaluate the correlation of GDF15 expression in the TME with the abundance of Treg cells. The clinicopathological characteristics of the patients revealed that GDF15 expression in the TME was positively correlated with the clinical stage (online supplemental table 2). We separated the 90 HCC samples into two cohorts by the mean frequency of Treg cells among $\mathrm{CD}^{+}$TILs (figure 1E) and validated GDF15 upregulation in tumors with a relatively high Treg cell frequency by ELISA (figure 1F) and IF staining (figure 1G). We also found that the concentration of GDF15 in tumors was positively associated with the frequencies of Treg cells in tumors (figure $1 \mathrm{H}$ and online supplemental figure 2A), draining lymph nodes (figure 1I) and peripheral blood in these patients wih HCG (figure 1J). To confirm our findings with additional data, we retrieved mRNA-seq data from The Cancer Genome Atlas (TCGA) datasets. We found that GDF15 mRNA expression was upregulated in HCC (figure 1K) and that high expression of GDF15 was significantly associated with shorter overall survival times (figure 1L). After normalization to CD4 mRNA expression, GDF15 mRNA expression in TCGA HCC samples was positively correlated with several Treg cell signature transcripts, such as CTLA4, TIGIT and TNFRSF18 or BATF (online supplemental figure 2B). Clustering these HCC tumor samples into two cohorts by unsupervised hierarchical clustering with a correlation matrix according to the mRNA expression levels of CD4 and CD25 (figure 1M) revealed that the cohort with high levels of both $C D 4$ and CD25 exhibited significantly increased GDF15 expression (figure 1N). Collectively, these data indicate that GDF15, as a detrimental factor in HCC, may play important roles in the generation or regulation of Treg cells.

\section{The immunosuppressive function of GDF15 in vivo is related to Treg cells}

To demonstrate that the detrimental effect of GDF15 on HCG is relevant to Treg cell-mediated 


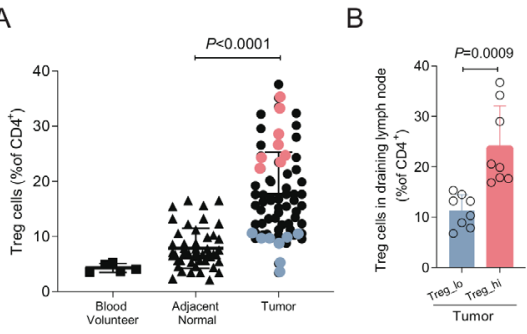

E

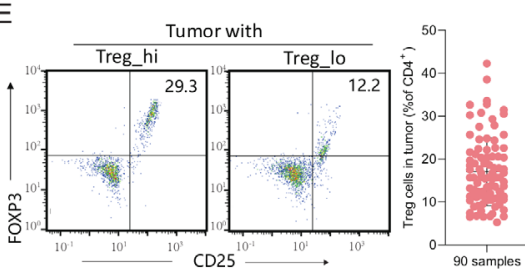

G
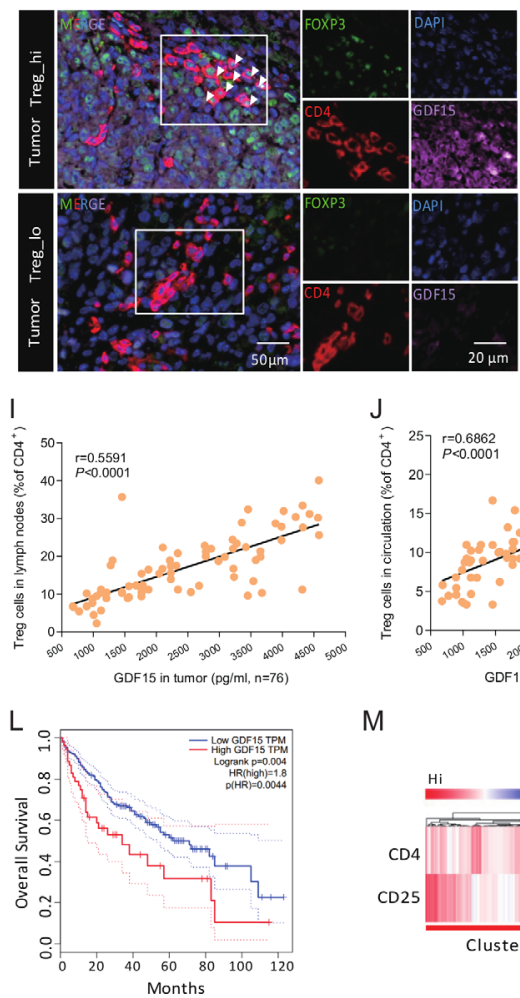

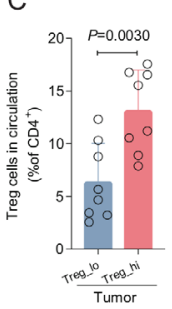

D
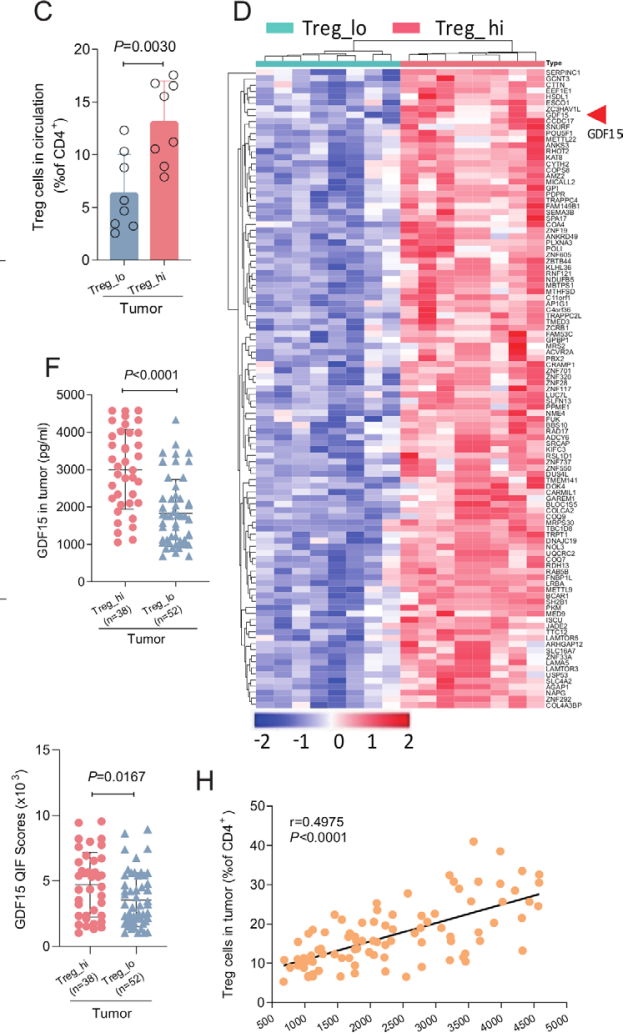

$\mathrm{H}$

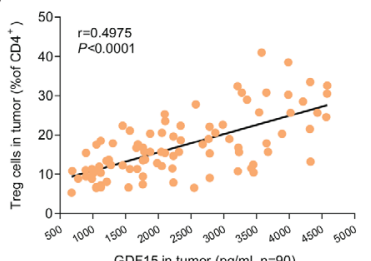

GDF15 in tumor (pg/mll, $n=90)$

K

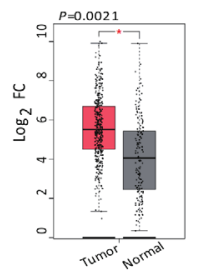

$\mathrm{N}$
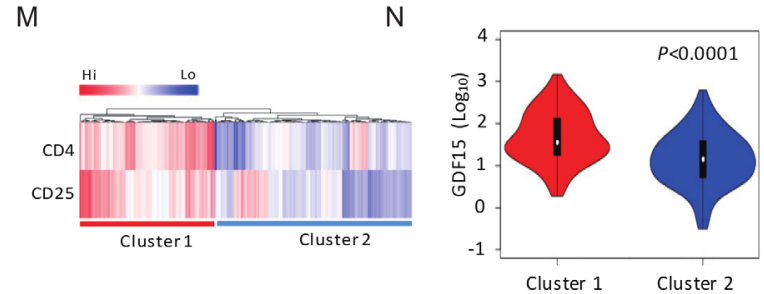

Figure 1 Identification of growth differentiation factor 15 (GDF15) as a modulator of regulatory $T$ (Treg) cells in hepatocellular carcinoma (HCC). (A) Treg cell frequencies among CD4 $4^{+} \mathrm{T}$ cells in $60 \mathrm{HCC}$ tissues, 48 normal liver tissues and 5 blood samples from volunteers. HCC tissues with a relatively high (red, Treg_hi) and low (blue, Treg_lo) Treg cell frequency were selected for RNA sequencing (RNA-seq) ( $n=8$ per group). (B, C) Treg cell frequency among CD4 ${ }^{+}$T cells in draining lymph nodes and peripheral blood of the 16 patients whose tissues were submitted for RNA-seq. (D) Heatmap of differentially expressed genes (fold change >2). (E-G) A $90 \mathrm{HCC}$ patient cohort was classified into two groups by the mean Treg cell frequency among CD4 ${ }^{+}$ tumor-infiltrating lymphocytes (TILs) $(17.04 \%, n=38$ vs $n=52)(E)$. GDF15 expression in TME was measured by ELISA (F) and quantitative immunofluorescence (GDF15 (purple), CD4 (red) and FOXP3 (green) and DAPI (blue)) (G). (H-J) The correlations between GDF15 concentrations and the frequencies of Treg cells in TME $(\mathrm{H})$, draining lymph nodes $(\mathrm{l})$ and peripheral blood $(\mathrm{J})$. (K) Messenger RNA (mRNA) expression levels of GDF15 in HCC versus corresponding normal tissues, as determined by metaanalysis of the The Cancer Genome Atlas (TCGA) database ( $n=367$ vs 149 samples of tumor vs normal tissues, respectively). (L) Kaplan-Meier survival curves for patients wih HCC with low and high GDF15 expression as determined by meta-analysis of the database. The cut-off value is the average GDF15 mRNA expression level (low GDF15 cohort, n=246; high GDF15 cohort, $\mathrm{n}=121$ ). The $95 \% \mathrm{Cls}$ are shown by dotted lines. TCGA data analysis is finished using GEPIA (gepia.cancer-pku.cn) online tool. (M, N) Heatmap for CD4 and CD25 expression of 367 patients in TCGA HCC cohort. Two clusters (1 and 2) were found by unsupervised hierarchical clustering with a correlation matrix on the basis of $C D 4$ and $C D 25$ expression levels (M). Relative GDF15 mRNA levels after normalization to CD4 mRNA levels in two clusters are shown (N). Data are representative of two independent experiments performed for the tissues isolated from each of patients $(B, C, E, F, H-J)$. $P$ values were determined by two-tailed unpaired t-test (A-C, F, G, N) or Pearson's correlation coefficient (H-J). 
immunosuppression, we first evaluated GDF15 expression in a spectrum of human (online supplemental figure 3A,B) and mouse HCC cell lines (online supplemental figure 3C). GDF15 was overexpressed in most of the HCC cell lines compared with normal liver cells. Neither knockout of GDF15 (online supplemental figure 3D-H and L) nor GDF15 addition (online supplemental figure 3I-K,M) affected the growth of human or mouse HCC cells in vitro. Similarly, knockout of GDF15 in Hepa1-6 mouse HCC cells did not affect their growth in the livers of Rag $T^{-}$ mice (online supplemental figure 4A). However, when Hepa1-6 or H22 mouse HCC cells was inoculated into the livers of normal syngeneic mice, knockout of GDF15 dramatically slowed tumor growth (figure $2 \mathrm{~A}$ and online supplemental figure 4B), prolonged the survival of the mice (figure 2B and online supplemental figure 4C) and significantly decreased the GDF15 concentrations in the circulation and TME of the mice to a physiological level (figure 2C and online supplemental figure 4D). Importantly, a markedly decreased frequency of Treg cells among $\mathrm{CD} 4^{+} \mathrm{T}$ cells was found in both the tumors and spleens of mice inoculated with GDF15 knockout cells (figure 2D and online supplemental figure 4E).

To evaluate overall immunological changes in tumors, we profiled TILs of Hepa1-6 GDF15 knockout and mock knockout tumors from normal syngeneic mice using mass cytometry (CyTOF), in which 42 monoclonal antibodies (mAbs) were used to determine immune cell lineages as well as functional molecules at a single cell dimension. The analysis of the $\mathrm{CD} 4^{+} \mathrm{T}$ cell population revealed a noticeable reduction in Treg cell frequencies in GDF15 knockout tumors (figure 2E,F and online supplemental figure 4F). The expression of Treg cell-related immunosuppressive molecules on $\mathrm{CD} 4^{+} \mathrm{T}$ cells of GDF15 knockout tumors was significantly decreased, while the expression of Ki67 was markedly upregulated (figure 2G). Further analysis of the total $\mathrm{CD} 45^{+}$hematopoietic cell population revealed 22 distinct cell subsets (clusters) (figure 2H,I and online supplemental figure $4 \mathrm{G}$ ). We found significant expansion of $\mathrm{CD}^{+} \mathrm{T}$ cells (cluster 2), $\mathrm{CD}^{+}$ effector/memory T cells (cluster 3 ) and $\mathrm{CD}^{3} 8^{-} \mathrm{BST}^{+} \mathrm{B}$ cells (cluster 9) in GDF15 knockout tumors (figure 2J), resembling the recently reported global impact of Treg cell deficiency on the cellular composition in the TME. ${ }^{14}$ Together, these observations suggest that the detrimental effect of GDF15 on HCC is associated with its immunosuppressive function, and Treg cells play important roles in mediating the immunosuppressive effects of GDF15.

\section{GDF15 efficiently converts naïve $\mathrm{CD}^{+} \mathrm{T}$ cells to iTreg cells in vitro}

Next, we aimed to acquire direct evidence to demonstrate the regulatory role of GDF15 on Treg cells. In vivo, Treg cells are generated either in the thymus (nTreg cells) ${ }^{6}$ or derived from the existing pool of naive $\mathrm{CD} 4^{+} \mathrm{T}$ cells in peripheral tissues ( $p$ Treg cells). In vitro, Treg cells derived from naïve $\mathrm{CD}^{+}{ }^{+} \mathrm{T}$ cells after $\mathrm{T}$ cell receptor (TCR) stimulation in the presence of TGF- $\beta$ and interleukin (IL)-2 are referred to as iTreg cells. ${ }^{27} 28$ The identity of GDF15 as a distant member of the TGF- $\beta$ superfamily prompted us to first determine its role in iTreg cell generation. To this end, we added GDF15 to an in vitro culture of human CD45RA ${ }^{+} \mathrm{CD} 25^{-}$naïve $\mathrm{CD} 4^{+} \mathrm{T}$ cells stimulated with antiCD3/anti-CD28-specific mAbs and IL-2 (online supplemental figure 5A). Similar to TGF- $\beta$, GDF15 induced a high frequency of $\mathrm{FOXP}^{+} \mathrm{CD} 25^{\text {hi }}$ Treg cells (figure $3 \mathrm{~A}$ ) and resulted in decreased proliferation of naïve $\mathrm{CD} 4^{+} \mathrm{T}$ cells (online supplemental figure 5B). Further analysis of GDF15-induced cells showed enhanced expression of key Treg cell-suppressive molecules, including CTLA4, TNFRSF4, TIGIT and GITR (figure 3B-E). Functionally, GDF15-induced iTreg cells showed strong suppressive activity against carboxy fluorescein succinimidyl ester (CFSE)-labeled human naïve $\mathrm{CD} 4^{+} \mathrm{CD} 25^{-} \mathrm{T}$ cells when cultured with anti-CD3/anti-CD28 antibodies and IL-2 in vitro (figure $3 \mathrm{~F}$ ). Like human naïve $\mathrm{CD} 4^{+} \mathrm{T}$ cells, Jurkat $\mathrm{T}$ cells were also induced to differentiate into iTreg cells in the presence of GDF15 (online supplemental figure 5C). Mouse GDF15 (mGDF15) and human GDF15 share $68.1 \%$ sequence identity and $84.1 \%$ sequence similarity (online supplemental figure 5D). Both mGDF15 (figure 3G,H) and human GDF15 (online supplemental figure $5 \mathrm{E}$ ) induced the differentiation of mouse naïve $\mathrm{CD}^{+} \mathrm{T}$ cells into iTreg cells, comparable to the effect of human GDF15 on human cells. Furthermore, we isolated naïve $\mathrm{CD} 4^{+} \mathrm{CD} 25^{-} \mathrm{T}$ cells from the spleens of $\mathrm{C} 57 \mathrm{BL} / 6$ green fluorescent protein (GFP) transgenic mice and cocultured them with mGDF15-induced iTreg cells from mouse naïve $\mathrm{CD}^{+} \mathrm{T}$ cells. Significant inhibitory effects of mGDF15-induced iTreg cells on these autofluorescent naïve $\mathrm{CD} 4^{+} \mathrm{T}$ cells was visually observed via real-time confocal microscopy (figure 3I, and online supplemental videos 1 and 2). These data support the hypothesis that GDF15 promotes efficient differentiation of naïve $\mathrm{CD} 4^{+} \mathrm{T}$ cells into $\mathrm{FOXP}^{+} \mathrm{CD}_{2} 5^{\text {hi }}$ iTreg cells in vitro.

\section{GDF15 but not TGF- $\beta$ substantially contributes to the inhibitory activity of $n$ Treg cells in vitro}

Some studies support that nTreg cells make a greater contribution to the intratumoral Treg compartment than iTreg cells, although the source of Treg cells in tumors remains controversial. ${ }^{6}$ To investigate whether GDF15 contributes to the plasticity of nTreg cells, we isolated $\mathrm{CD} 44^{+} \mathrm{CD} 25^{\mathrm{hi}} \mathrm{CD} 127^{\text {lo }}$ primary nTreg cells from human peripheral blood or mouse spleens as described in previous studies. ${ }^{27} 29$ Then, we assessed the effects of GDF15 on the expansion and function of nTreg cells in vitro. Neither human GDF15 nor mGDF15 effectively increased the proliferation of human or mouse nTreg cells in the presence of anti-CD3/anti-CD28 antibodies and IL-2 in vitro (online supplemental figure 6). However, GDF15 and mGDF15 noticeably elevated the expression levels of FOXP3 (online supplemental figure 7A,C) and cytotoxic T lymphocyte-associated molecule 4 (CTLA4) (online supplemental figure 7B,D) in the corresponding human or mouse nTreg cells. Unlike GDF15, 

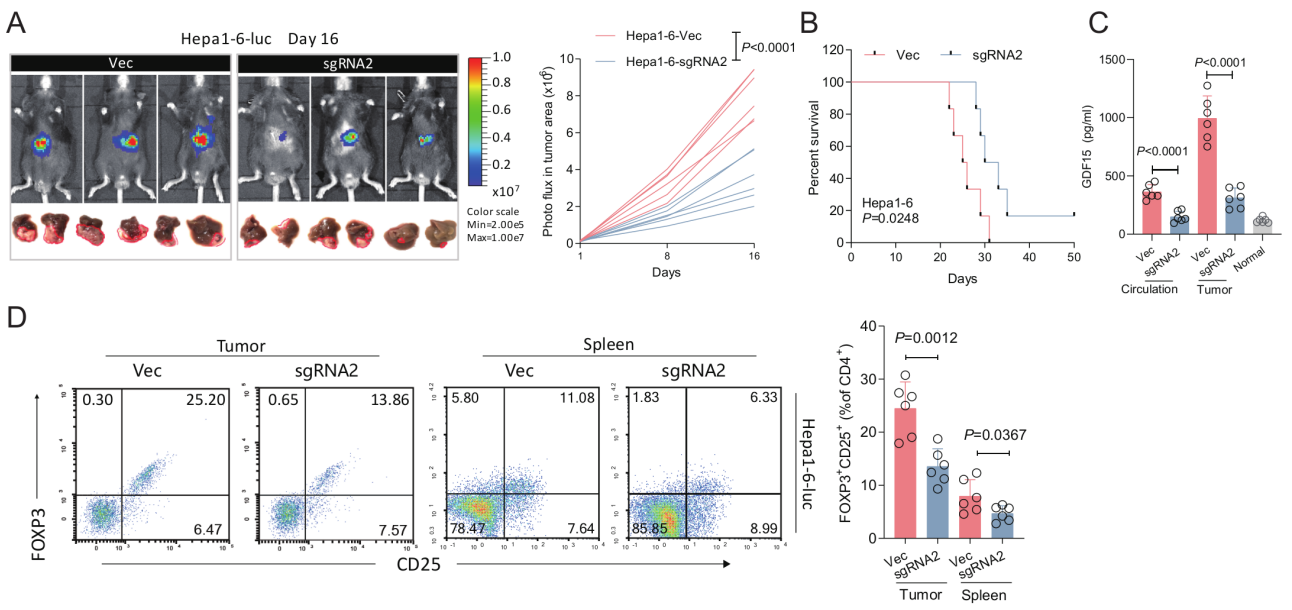

E

$E_{\text {CD4 clusters }}$

G
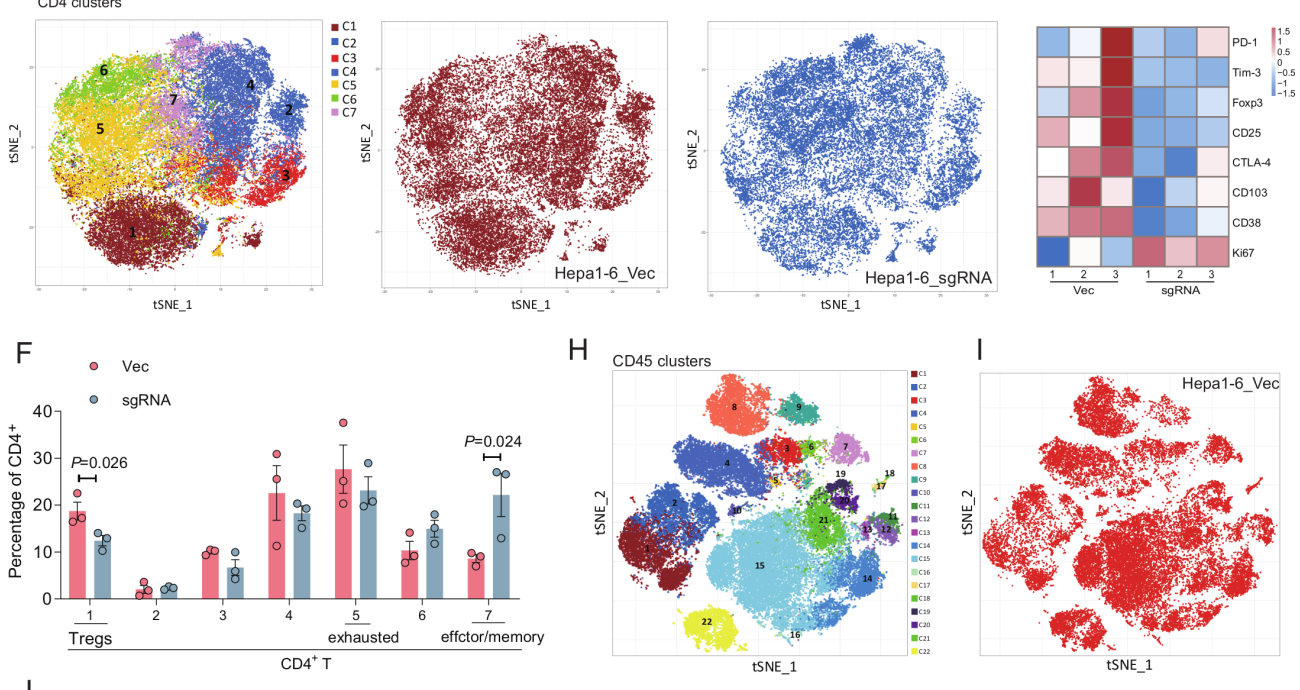

$\mathrm{H}$ CD45 clusters
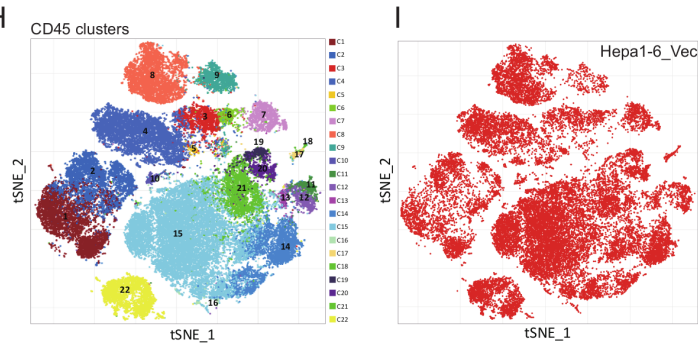

$J$
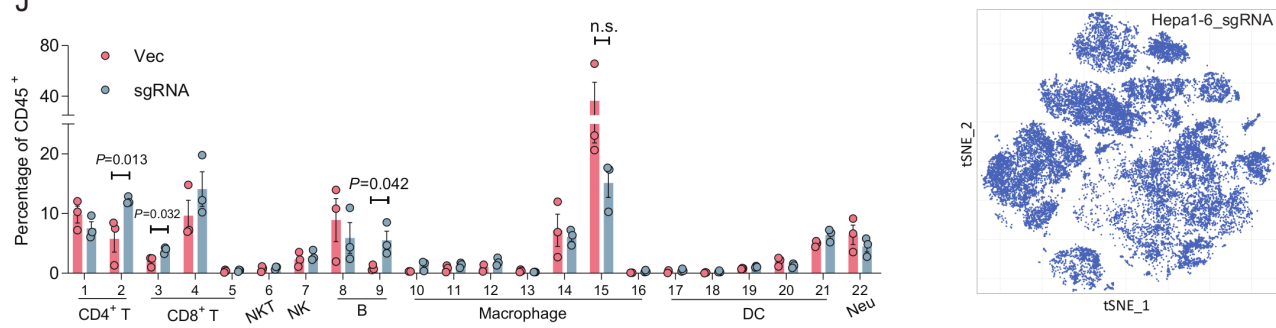

Figure 2 The immunosuppressive function of growth differentiation factor 15 (GDF15) in vivo is related to regulatory T (Treg) cells. (A-D) GDF15 knockout Hepa1-6-luc cells (sgRNA2) or mock cells (Vec) were inoculated into the livers of syngeneic C57BL/6 mice ( $n=6$ mice per group). Tumor growth was monitored by values of bioluminescence $\left(\mathrm{p} / \mathrm{s} / \mathrm{cm}^{2} / \mathrm{sr}\right)(A, \mathrm{right})$. Representative bioluminescence imaging of three mice in each group on day 16 and the liver images of all mice acquired on day 28 after euthanasia ( $A$, left; the tumor is identified by the red line) are shown. Survival was evaluated by using another two groups of mice ( $n=6$ mice per group) (B). GDF15 concentrations in the tumor and circulation of mice on day 28 after euthanasia $(C)$ and the frequencies of Treg cells among CD4 ${ }^{+}$tumor-infiltrating lymphocytes (TILs) and splenic cells (D) were analyzed. (E-J) Sixteen days after Hepa1-6-sgRNA or Hepa1-6-Vec cells inoculation (n=9 mice per group), the tumor of each mouse was digested and filtered to obtain a single cell suspension. The cell suspensions of every three mice in each group were pooled. CD $45^{+}$tumor-infiltrating leukocytes were analyzed by mass cytometry (CyTOF) ( $n=3$ samples per group). The $t$-distributed stochastic neighbor embedding ( $t$-SNE) plot of CD4 ${ }^{+}$tumor-infiltrating leukocytes of total six samples (pooled data) (E, left panel; overlaid with color-coded clusters) and density $t$-SNE plots of CD4 ${ }^{+}$tumor-infiltrating leukocytes in Hepa16-sgRNA or Hepa1-6-Vec group (E, right two panels; $2 \times 10^{5}$ cells per group were displayed) are shown. The frequencies of the $\mathrm{CD}^{+}$clusters were calculated as the assigned cell events divided by the total $\mathrm{CD}^{+}$cell events in the same sample (F). The normalized expression values (mean mass intensities) of Treg cell-related molecules and Ki67 in the CD4 ${ }^{+}$population are shown as a heatmap $(\mathrm{G})$. The $\mathrm{CD} 45^{+}$population was plotted by $t$-SNE and density $t$-SNE with same methods, and it overlaid with 22 clusters $(\mathrm{H}, \mathrm{I})$. The frequencies of the indicated immune cell subsets within the CD45 ${ }^{+}$population are shown $(\mathrm{J})$. Data are representative of three independent experiments $(A-D)$. $P$ values were determined by a two-tailed Mann-Whitney $U$ test $(A)$, a log rank test $(B)$ and a two-tailed unpaired t-test (C, D, F, J). n.s., not significant; $p>0.05$. 
A

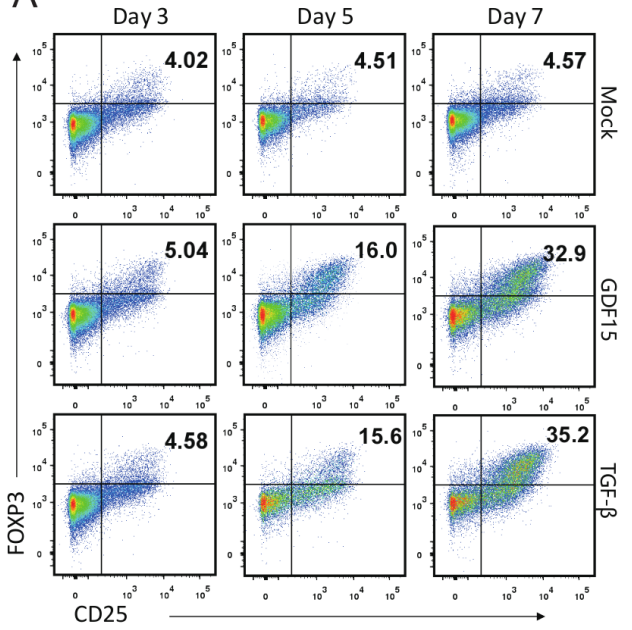

B

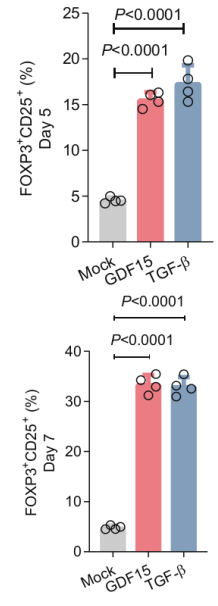

C
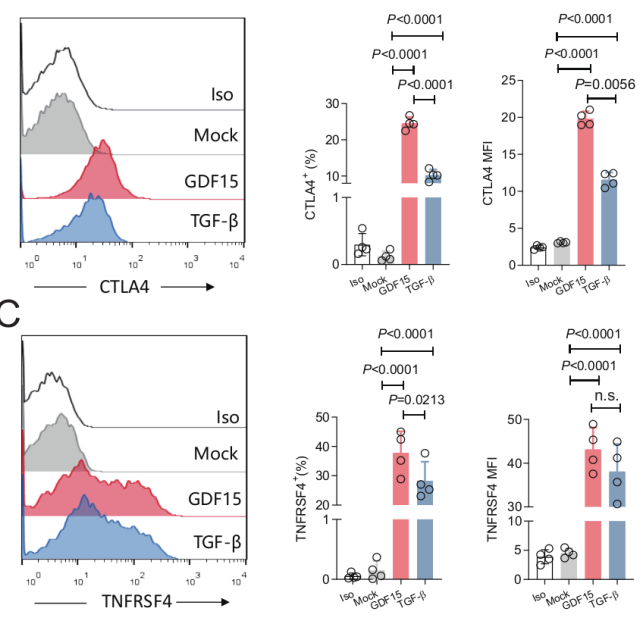
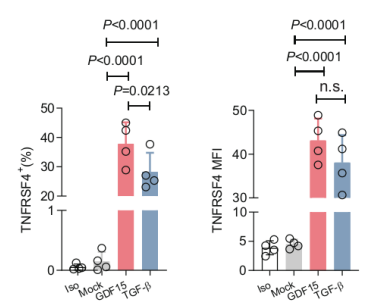

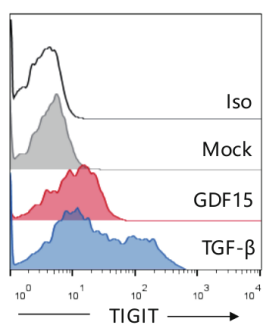

F

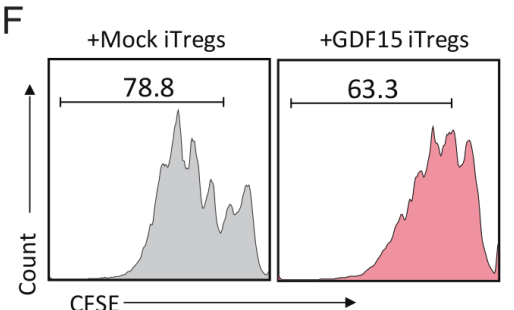

$\mathrm{H}$

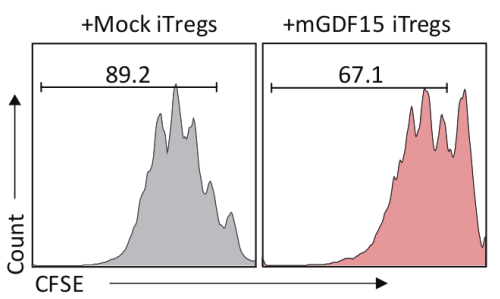

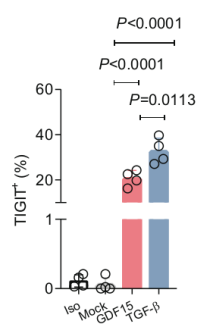

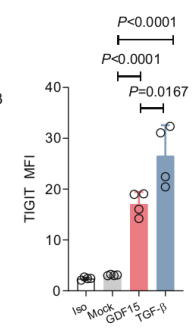

E

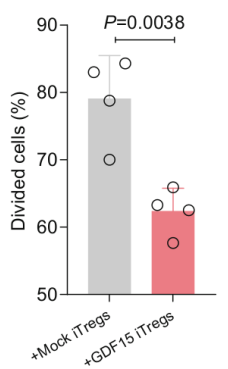

G

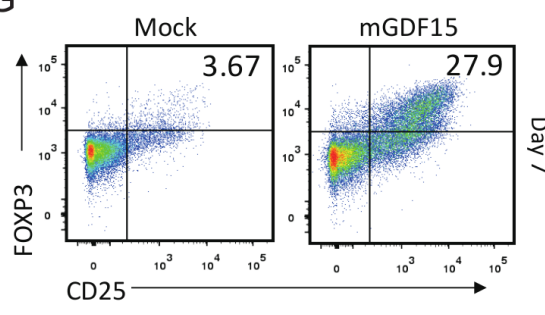

I

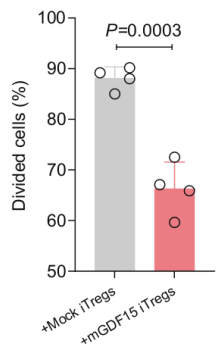

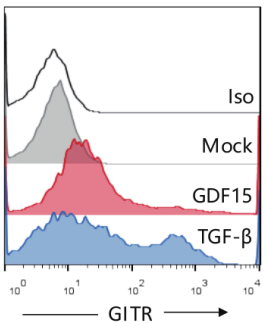
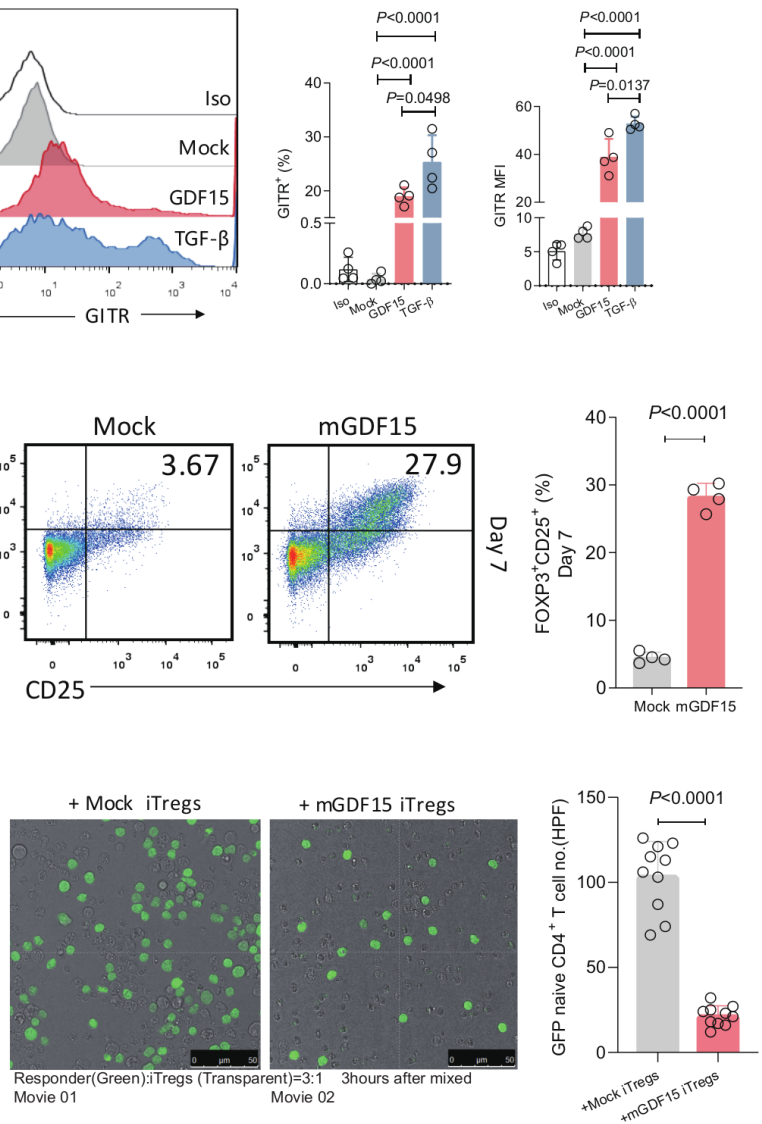

Figure 3 Growth differentiation factor 15 (GDF15) induces the generation of inducible regulatory $\mathrm{T}$ (iTreg) cells in vitro with an effect comparable to that of transforming growth factor $\beta$ (TGF- $\beta$ ). (A-E) The iTreg cell generation from human naïve CD4 ${ }^{+}$ cells after GDF15, TGF- $\beta$ or Mock conversion (A). The expression of Treg cell signature genes (CTLA4, TNFRSF4, TIGIT, GITR) of the converted $C D 4^{+} T$ cells $(B-E)$. (F) The inhibition of iTreg cells induced from human naïve CD4 ${ }^{+}$T cells by GDF15 (GDF15 iTregs) or mock treatment (Mock iTregs) to the proliferation of naïve $\mathrm{CD} 4^{+} \mathrm{CD} 25^{-} \mathrm{T}$ cells was determined by carboxy fluorescein succinimidyl ester (CFSE) dilution assay ( $n=4$ cell cultures from four healthy donors). (G, $H$ ) The iTreg cell generation from mouse splenic naïve $C D 4^{+} T$ cells after mouse GDF15 (mGDF15) or mock conversion (G). The inhibition of iTreg cells induced from mouse naïve $\mathrm{CD}^{+} \mathrm{T}$ cells by mouse GDF15 (mGDF15 iTregs) or mock treatment (Mock iTregs) to the proliferation of mouse naïve $\mathrm{CD}^{+} \mathrm{T}$ cells was determined by CFSE dilution assay (H). (I) The inhibitions of iTreg cells induced from mouse naïve $\mathrm{CD}^{+} \mathrm{T}$ cells (transparent) by mouse GDF15 (mGDF15 iTregs) or mock treatment (Mock iTregs) to GFP CD4 ${ }^{+} \mathrm{T}$ cell was visually observed with real-time confocal microscopy for 8 hours (see online supplemental videos 1 and 2). Live GFP CD4 ${ }^{+}$T cells were counted 3 hours after mixing (for each cell culture, 10 high-power fields (HPFs) were counted) ( $n=4$ cell cultures). Mock, antiCD3/anti-CD28 monoclonal antibodies (mAbs) and interleukin (IL)-2 stimulations. Data are representative of three independent experiments. $P$ values were determined by a two-tailed unpaired t-test. Iso, isotype control; n.s., not significant; $p>0.05$. 
TGF- $\beta$ did not exhibit similar effects. Corroborating the promotive effect of GDF15 on the inhibitory molecules of nTreg cells, the suppressive activity of human or mouse nTreg cells against the corresponding CFSE-labeled naive $\mathrm{CD} 4^{+} \mathrm{CD} 25^{-} \mathrm{T}$ cells were noticeably enhanced after GDF15 exposure (online supplemental figure 7E,F). Moreover, we cocultured mGDF15-treated mouse nTreg cells with $\mathrm{CD}^{+} \mathrm{T}$ cells obtained from OT-I TCR transgenic mice (OT-I T cells) and Hepa1-6-OVA cells. Hepa1-6-OVA is a mouse HCC cell line stably transfected with chicken ovalbumin (OVA), which allows antigen-specific cytotoxicity to $\mathrm{OVA}_{257-264}$ peptide-activated OT-I T cells. mGDF15treated mouse nTreg cells demonstrated superior suppressive activity against OT-I T cells compared with untreated mouse nTreg cells in the xCELLigence real-time cellular impedance assay (RTCA) (online supplemental figure $7 \mathrm{G}$ ). These results indicate that unlike TGF- $\beta$, GDF15 facilitates the generation of iTreg cells, and enhances the suppressive function of nTreg cells.

\section{GDF15-deficient mice are defective in the generation of competent Treg cells}

To verify our in vitro findings, we generated a GDF15 whole-body knockout mouse strain (GDF15 KO) and confirmed the absence of GDF15 in serum and various organs (online supplemental figure 8A). Knockout of GDF15 did not affect the viability of fetal mice. At 10 months, GDF15 KO mice did not display obvious disease phenotypes as evaluated by pathological scoring of the inflammatory status in the main organs (online supplemental figure 8B). However, GDF15 KO mice produced few HCC nodules in liver when a transposon system $^{30}$ containing the plasmids encoding myr-AKT1 and N-RasV12 along with sleeping beauty transposase was delivered to hepatocytes via hydrodynamic tail vein injection to induce orthotopic HCC (online supplemental figure 8C). The intact GDF15 $\mathrm{KO}$ mice had significant lower frequency of $\mathrm{FOXP3}^{+} \mathrm{CD} 25^{\text {hi }}$ Treg cells among $\mathrm{CD}^{+} \mathrm{T}$ cells in peripheral organs, including the spleen, mesenteric lymph nodes (MLNs), gut lamina propria (gLP) and blood than their wild-type (WT) littermates. The percentage of Treg cells in the thymus, the source of nTreg cells in vivo, did not noticeably differ between GDF15 KO mice and their WT littermates (figure 4A). In addition, $\mathrm{CD} 4^{+} \mathrm{T}$ cells isolated from the spleens of GDF15 $\mathrm{KO}$ mice exhibited an activated phenotype with more $\mathrm{CD} 4^{+} \mathrm{CD} 62 \mathrm{~L}^{\text {lo }} \mathrm{CD} 44^{\text {hi }}$ (figure 4B) and interferon- $\gamma$ (IFN$\gamma)^{+}$cells (online supplemental figure 8D) than those from the WT littermates. When Treg cells from GDF15 KO or WT mice were cocultured with Hepa1-6-OVA cells and OT-I T cells, Treg cells from WT littermate mice demonstrated superior suppressive activity against OT-I T cells compared with Treg cells from GDF15 KO mice in the RTCA (figure 4C).

To further elegantly demonstrate the effects of GDF15 on pTreg and nTreg cells in vivo, we transferred same amount of naive $\mathrm{CD} 4^{+} \mathrm{T}$ cells from C57BL/6 GFP transgenic mice (B6-GFP (CD45.1)) into irradiated GDF15 KO
(CD45.2) mice or their WT littermates (online supplemental figure 8E). Five days later, fewer CD45.1 FOXP3 ${ }^{+}$ Treg cells were detected in the spleens of KO mice than in those of their WT littermates (figure 4D). Then, we transferred primary nTreg cells from the spleens of B6-GFP (CD45.1) mice into GDF15 KO mice or their WT littermates (online supplemental figure 8F). Five days later, the numbers of CD45.1 Treg cells in the spleens of GDF15 KO mice and their WT littermates were comparable (figure 4E). However, FOXP3 and CTLA4, molecules related to Treg cell functions, were markedly upregulated in CD45.1 Treg cells recovered from WT littermate mice compared with those recovered from GDF15 KO mice (figure 4F,G). These data are consistent with the in vitro effects of GDF15 on the efficient generation of iTreg cells and the functional enhancement of nTreg cells.

\section{GDF15 blocks the ubiquitination of FOXP3}

In the aforementioned study, we proved by flow cytometry that GDF15, like TGF- $\beta$, noticeably enhanced the protein expression of FOXP3 under iTreg cell skewing conditions (figure 3A). However, further investigation showed that although GDF15 increased the protein expression of FOXP3 in human and murine naive $\mathrm{CD}^{+} \mathrm{T}$ cells (figure 5A), it did not affect FOXP3 mRNA expression (figure 5B), unlike TGF- $\beta$. Additionally, when protein synthesis was blocked by cycloheximide (CHX), exposure to GDF15 but not TGF- $\beta$ markedly increased the stability of FOXP3 in human and murine naïve $\mathrm{CD} 4^{+}$ $\mathrm{T}$ cells (figure 5C). Moreover, the slightly increased FOXP3 level on stimulation with anti-CD28/anti-CD3 mAbs and IL-2 (mock induction in figure 5A,B), which is a widely reported upregulation without cell phenotype changes, ${ }^{31-33}$ suggests that GDF15 causes FOXP3 accumulation by inhibiting its degradation based on TCR activation.

Polyubiquitination is a pivotal way of protein degradation. We then sought to determine whether GDF15induced accumulation of FOXP3 depends on blockade of ubiquitination. Indeed, GDF15 but not TGF- $\beta$ obviously decreased FOXP3 ubiquitination in human (figure 5D) and mouse (figure $5 \mathrm{E}$ ) naïve $\mathrm{CD} 4^{+} \mathrm{T}$ cells, as shown by coimmunoprecipitation (CoIP). Reciprocal CoIP with an antibody against ubiquitin (Ub) also confirmed that GDF15 decreased FOXP3 ubiquitination in human naïve $\mathrm{T}$ cells (figure $5 \mathrm{~F}$ ). More importantly, GDF15 decreased FOXP3 ubiquitination in both human (figure 5G) and mouse (figure $5 \mathrm{H}$ ) primary nTreg cells stimulated with antihuman and antimouse CD28/CD3 mAbs, respectively, and IL-2. We further performed RNA-seq on GDF15-induced and TGF- $\beta$-induced iTreg cells derived from human naive $\mathrm{CD} 4^{+} \mathrm{T}$ cells. The gene set enrichment analysis (GSEA) (figure 5I and online supplemental figure 9A) and GO analysis (online supplemental figure 9B) revealed enrichment of ubiquitination-related genes among the differentially expressed genes between the two kinds of iTreg cells. These data indicate that blocking FOXP3 ubiquitination is the mechanism by which GDF15 
A
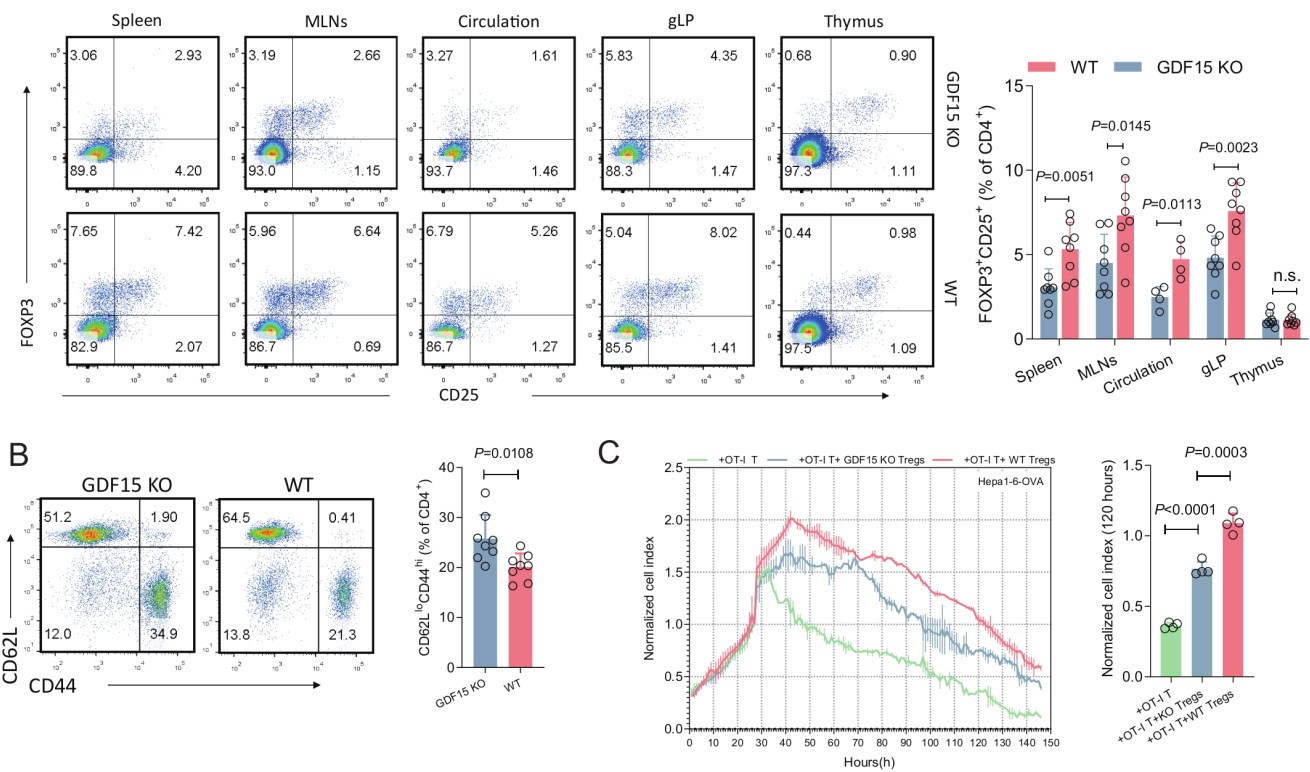

D

GDF15 KO

WT
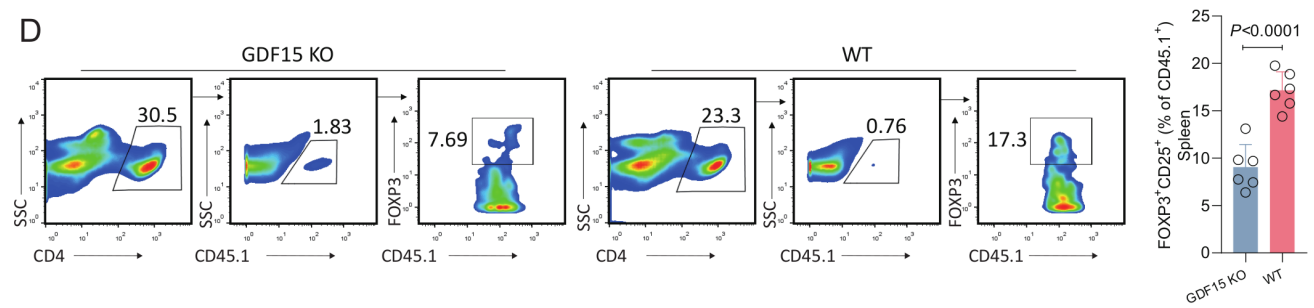

E
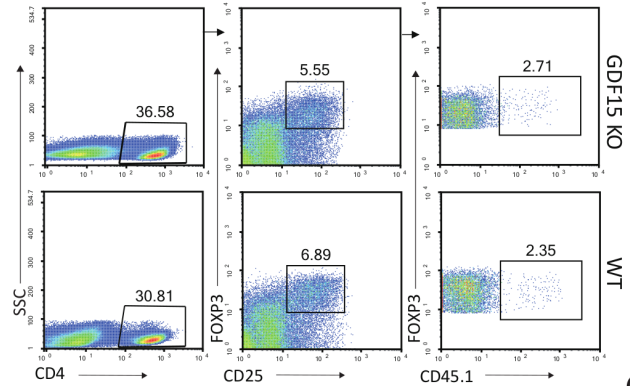

F
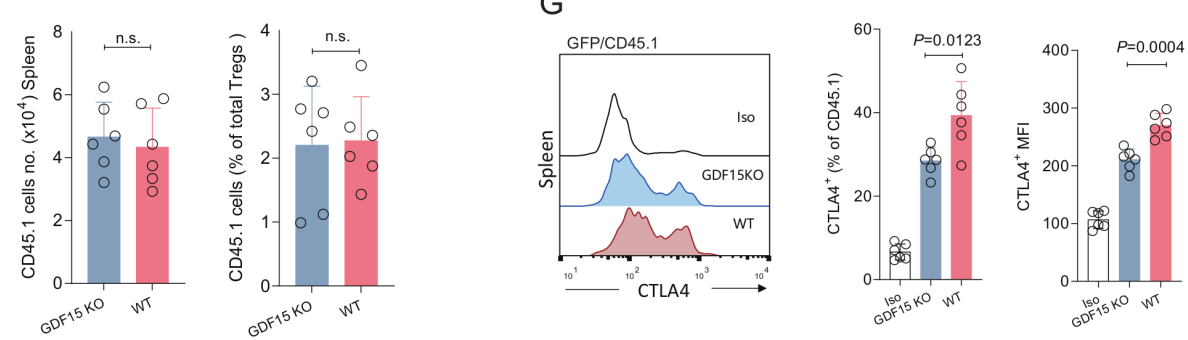

Figure 4 Growth differentiation factor 15 (GDF15)-deficient mice are defective in the generation of competent regulatory $\mathrm{T}$ (Treg) cells. (A, B) Frequencies of Treg cells among the total CD4 ${ }^{+} \mathrm{T}$ cell population in spleen, mesenteric lymph nodes (MLNs), gut lamina propria (gLP), peripheral blood and thymus of GDF15 whole-body knockout mouse strain (GDF15 KO) or wild-type (WT) mice (A). The activation status of splenic CD4 ${ }^{+}$T cells of GDF15 KO or WT mice (B) ( $n=8$ mice per group). (C) Real-time survival of Hepa1-6-OVA cells cocultured with OT-I cells (+OTI), OT-I cells plus Treg cells from GDF15 KO mice (+OTI+GDF15KO Tregs) or from WT mice (+OTI+WT Tregs). Data at 120 hours are shown as a bar ( $n=4$ cell cultures). (D) A total of $2 \times 10^{6}$ naive CD4 ${ }^{+}$T cells from B6-GFP (CD45.1) mice were transferred into irradiated GDF15 KO or WT mice ( $\mathrm{n}=6$ mice per group). Five days later, CD45.1 T cells of GDF15 KO or WT mice were sorted and the frequency of Treg cells was determined. (E-G) A total of $2 \times 10^{6}$ primary natural Treg (nTreg) cells of B6-GFP (CD45.1) mice were transferred into irradiated GDF15 KO mice or their WT littermates ( $n=6$ mice per group). Five days later, CD $4^{+} C D 25^{\text {hi }}$ FOXP $3^{+}$Treg cells were isolated, and the quantity and proportion of CD45.1 cells among the isolated Treg cells (E), and the expression of forkhead box P3 (FOXP3) and cytotoxic T lymphocyte-associated molecule 4 (CTLA4) in sorted CD45.1 cells was determined (F, G). Data are representative of two (A, B) or three $(\mathrm{C}-\mathrm{G})$ independent experiments. $\mathrm{P}$ values were determined by two-tailed unpaired t-test. Iso, isotype control; n.s., not significant; $p>0.05$. 
A

B
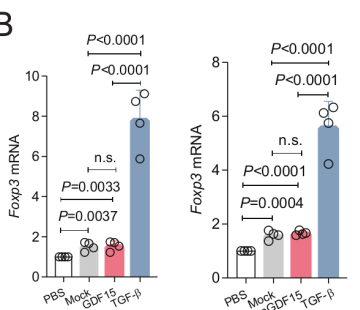

C

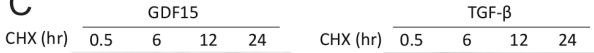
FOXP3 $-2 . \quad$ FOXP3 $-\div$ $\begin{array}{llll}1.00 & 0.89 & 0.56 \quad 0.26 \\ - & -\end{array}$ \begin{tabular}{llll}
1.00 & 1.56 & 0.12 & 0.09 \\
\hline
\end{tabular}

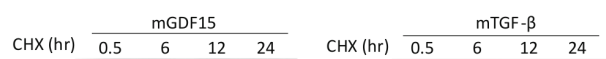

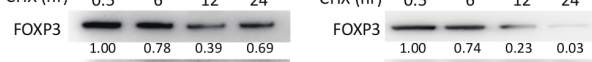
GAPDH $\longrightarrow-$ GAPDH $\stackrel{1.00}{-0.74}=0.23$

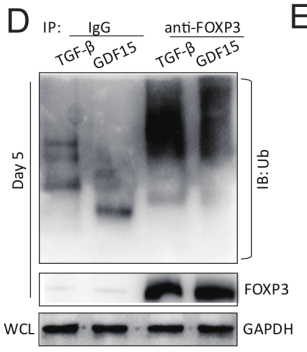

E
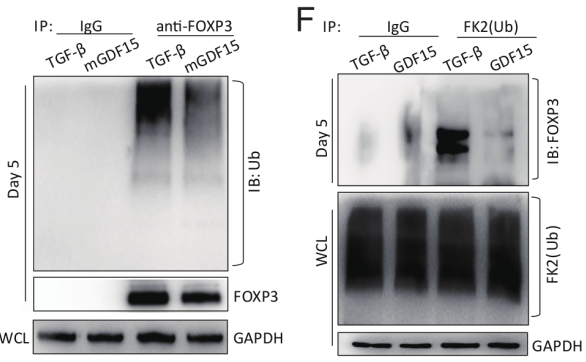

I

G

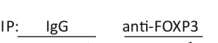

$\mathrm{H}$

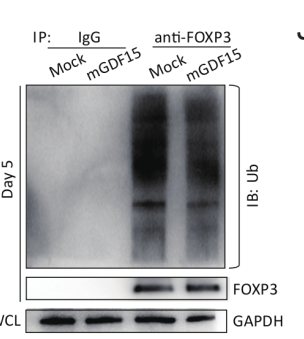

J
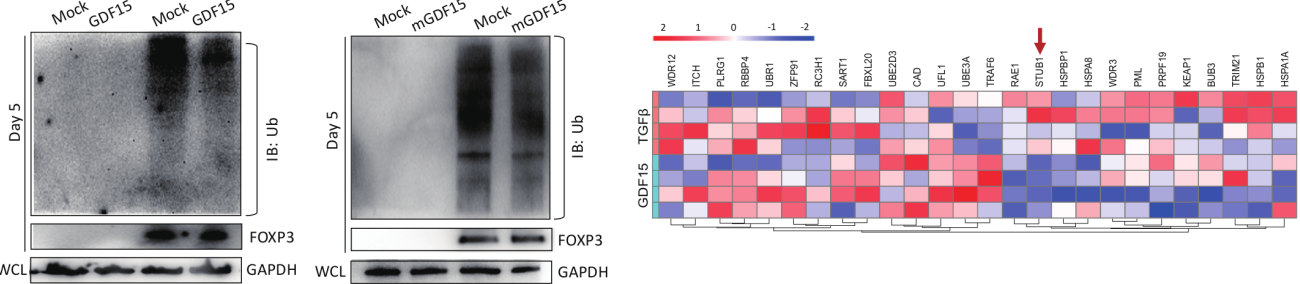

K

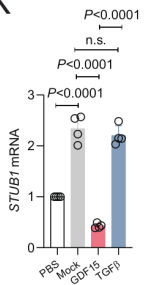

$\mathrm{N}$

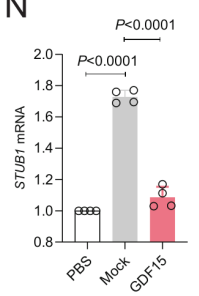

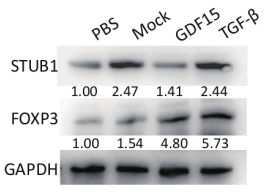

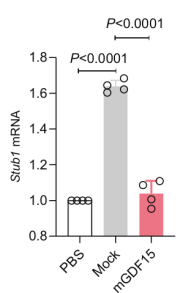

L

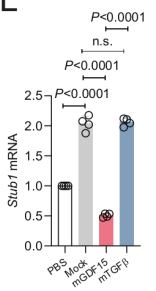

0

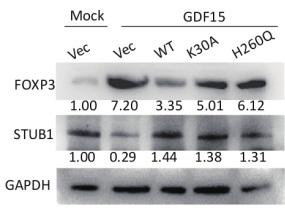

M

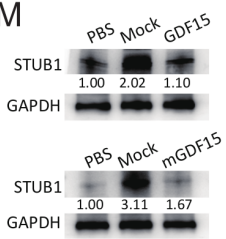

P

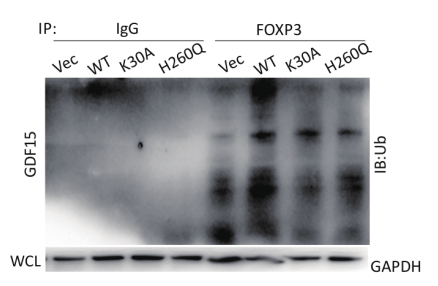

Figure 5 Growth differentiation factor 15 (GDF15) blocks FOXP3 ubiquitination by downregulating STUB1. (A, B) The protein and messenger RNA (mRNA) expressions of FOXP3 in human or mouse naïve CD4 ${ }^{+}$T cells after indicated stimulation. (C) FOXP3 expressions in GDF15 or transforming growth factor $\beta$ (TGF- $\beta$ ) induced human (upper panel) or mouse (lower panel) inducible regulatory $T$ (iTreg) cells after treated with cycloheximide (CHX) of the indicated time. (D, E) Coimmunoprecipitation (ColP) analysis of FOXP3 ubiquitination in human (D) and mouse (E) naïve CD4 ${ }^{+} T$ cells after indicated stimulation. (F) Reciprocal ColP analysis of FOXP3 ubiquitination in human naïve CD4 $4^{+} \mathrm{T}$ cells. $(\mathrm{G}, \mathrm{H})$ ColP analysis of FOXP3 ubiquitination in human (G) and mouse (H) primary natural Treg (nTreg) cells after indicated stimulation. (I) Gene set enrichment analysis (GSEA) of ubiquitination genes in GDF15 converted iTreg cells versus those in TGF- $\beta$ converted iTreg cells. The enrichment score (ES) and $p$ value are reported for the gene ontology (GO) term 'protein ubiquitination' and were calculated from GSEA data with weighted enrichment statistics and the ratio of classes for the metric as input parameters. (J) Heatmap showing expressions of 26 FOXP3 ubiquitination-related genes (including STUB1, arrow) in GDF15 or TGF- $\beta$ converted iTreg cells ( $n=4$ cell cultures). $(K, L)$ The expression of STUB1 in GDF15 or TGF- $\beta$ converted iTreg cells from human (K) and mouse $(L)$ naïve CD4 ${ }^{+} T$ cells. $(\mathrm{M}, \mathrm{N})$ The protein $(\mathrm{M})$ and mRNA (N) expression levels of STUB1 in GDF15 simulated human nTreg cells or mouse GDF15 simulated mouse nTreg cells. (O, P) FOXP3 accumulation (O) and ubiquitination (P) in either STUB1 (WT) or its K30A or H260Q mutants or control vector $(\mathrm{Vec})$ successfully transduced Jurkat T cells after indicated stimulation. Mock, anti-CD3/anti-CD28 monoclonal antibodies (mAbs) and interleukin (IL)-2 stimulations. Data are representative of three independent experiments. The mRNA expressions of FOXP3 and STUB1 are normalized to that of the reference gene GAPDH (B, K, L, N, $\mathrm{n}=4$ cell culture). The intensities of target bands were quantified and normalized to GAPDH expression ( $, C, K-M, O)$. P values were determined by a two-tailed unpaired t-test (B, K, L, N), n.s., not significant; WCL, whole-cell lysate; $p>0.05$. 
induces the generation of iTreg cells and enhances the suppressive functions of nTreg cells, which is completely distinct from the transcriptional regulation mechanism of TGF- $\beta$.

\section{STUB1 is a pivotal E3 ubiquitin ligase that mediates the inhibitory effect of GDF15 on FOXP3 ubiquitination}

We then used an anti-FOXP3 antibody to pull down its associated binding partners and performed mass spectrometry to identify the potential E3 Ub ligase of FOXP3 in human Treg cells. Twenty-five E3 Ub ligases and E3 ligase-related proteins that specifically bound to FOXP3 but not to the IgG control were identified (figure 5J and online supplemental table 3). This list contained HSPA1A, a $70 \mathrm{kDa}$ heat shock protein that can recruit the stress-activated E3 Ub ligase STUB1 to FOXP3 for degradation. ${ }^{29}$ RNA-seq analysis of GDF15-induced or TGF- $\beta$-induced iTreg cells showed that GDF15 but not TGF- $\beta$ significantly downregulated the mRNA expression of STUB1 (figure 5J). Therefore, we focused on STUB1 in our subsequent experiments because of its identified negative modulation of FOXP3. ${ }^{29}{ }^{34}$ qRT-PCR analysis confirmed that $S T U B 1$ was upregulated by TCR activation (mock) and downregulated by GDF15 but not TGF- $\beta$ in both human (figure 5K) and mouse (figure 5L) naive $\mathrm{CD}^{+} \mathrm{T}$ cells. Consistently, STUB1 protein expression was downregulated after GDF15 stimulation, and FOXP3 expression was upregulated (figure $5 \mathrm{~K}$ and $\mathrm{L}$ ). We also observed a decrease in STUB1 on GDF15 stimulation in human and mouse primary nTreg cells (figure $5 \mathrm{M}$ and $\mathrm{N})$.

In Jurkat $\mathrm{T}$ cells, we repeatedly observed the effects of GDF15 including inhibitions to the degradation and ubiquitination of FOXP3 (online supplemental figure 9C-E) and downregulation to STUB1 in a dosedependent manner (online supplemental figure 9F,G). Moreover, the ectopic expression of STUB1 disrupted the GDF15-induced accumulation of FOXP3 (figure 5O) and substantially restored FOXP3 ubiquitination in Jurkat $\mathrm{T}$ cells (figure 5P), while mutants of STUB1 deficient in Hsp70 chaperone binding (K30A) or enzymatic activity (H260Q) failed to abrogate the effects of GDF15 on FOXP3. All the data suggest that GDF15 blocks FOXP3 ubiquitination by downregulating STUB1 at the transcriptional level.

\section{GDF15 interacts with CD48 and downregulates STUB1 through inhibition of the ERK/AP-1 pathway}

At present, glial-cell-derived neurotrophic factor family receptor $\alpha$-like (GFRAL) is the only confirmed receptor of GDF15. However, the expression of GFRAL is highly restricted in the neuronal cells of the hindbrain and is virtually absent in all of peripheral tissues ${ }^{19-21}$ In Jurkat, naive human and mouse $\mathrm{CD} 4^{+} \mathrm{T}$ cells, neither GFRAL nor its coreceptor RET were observed (online supplemental figure 10A,B). To understand the mechanism underlying STUB1 downregulation in response to GDF15 stimulation, we used His-tag-mediated pull-down assays to identify the receptor protein of GDF15 on Jurkat T cells. Subsequent mass spectrometry sequencing revealed that CD48 (UniProtKB: P09326) was the only membrane protein among the precipitated molecules (online supplemental figure 10C and table 4). CoIP (figure 6A) and ELISA (figure 6B) confirmed the interaction between GDF15 and CD48. ELISA also proved TGF- $\beta$ does not interact with CD48. Moreover, the interaction of GDF15 with plate-bound CD48 was blocked by the addition of soluble CD48 protein (figure 6C). When the expression of CD48 was knocked out, the binding of GDF15 on Jurkat T cells significantly diminished (figure 6D). The GDF15-CD48 interaction had a $\mathrm{Kd}$ of $\sim 0.1 \mu \mathrm{M}$, as measured by surface plasmon resonance (SPR) (figure 6E). Similarly, mGDF15 also interacted with mouse CD48 in a dose-dependent manner according to ELISA (figure 6F).

The propagation of TCR signaling on TCR engagement in $\mathrm{CD}^{+} \mathrm{T}$ cells include several downstream cascades. ${ }^{35}$ As a costimulatory factor, CD48 activates intracellular lymphocyte-specific protein tyrosine kinase (Lck) and downstream ERK pathway of TCR signaling after participating into the TCR complex. ${ }^{36}{ }^{37}$ In human (figure 6G and online supplemental figure 11A) and mouse naïve $\mathrm{CD}^{+} \mathrm{T}$ cells (figure $6 \mathrm{H}$ and online supplemental figure 11B), we observed that GDF15 treatment led to a steady decline in the phosphorylation of Lck and ERK. Activator protein (AP-1), which is composed of heterodimers and homodimers of c-jun and c-fos family members, is the main downstream effectors regulated by ERK. ${ }^{38}$ In GDF15-induced human (figure 6I) and mouse (figure 6J) iTreg cells and GDF15-treated human and mouse nTreg cells (online supplemental figure 11C), we observed decreasing levels of c-jun and c-fos proteins in the nuclear fraction. In the aforementioned study, we observed STUB1 was upregulated by TCR activation and GDF15 abrogated this upregulation significantly (figure $5 \mathrm{~K}$ and L). Additionally, GDF15 did not change TCR induced slight but significant upregulation of FOXP3 mRNA (mock and GDF15 treatment in figure 5B). We therefore performed promoter analysis in the JASPAR CORE database. The prediction results demonstrated that the promoter of STUB1 (online supplemental figure 11D) but not the promoter of FOXP3 has the binding sites of c-jun/c-fos. Dual-luciferase reporter assays showed that c-jun/c-fos significantly upregulated STUB1 promoter activity but had no regulation on FOXP3 promoter (online supplemental figure 11E,F). With chromatin immunoprecipitation experiments, we showed that the binding of c-jun/c-fos to the STUB1 promoter in either human and mouse iTreg cells (figure $6 \mathrm{~K}$ and $\mathrm{L}$ ) or human and mouse nTreg cells (online supplemental figure 11G,H) drastically decreased after GDF15 treatment.

In Jurkat T cells, GDF15 also led to a decline in the phosphorylation of ERK (online supplemental figure 11I). More importantly, the ability of GDF15 to inhibit the phosphorylation of ERK, inhibit the expression of STUB1 and increase the accumulation of FOXP3 in Jurkat T cells was diminished by CD48 transfection (figure 6M). CD48 
A

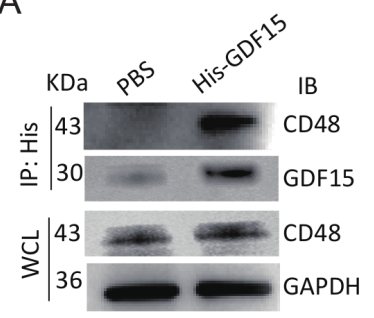

D

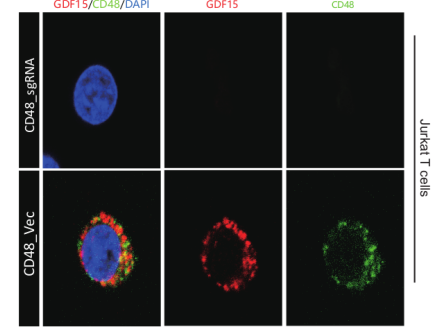

G

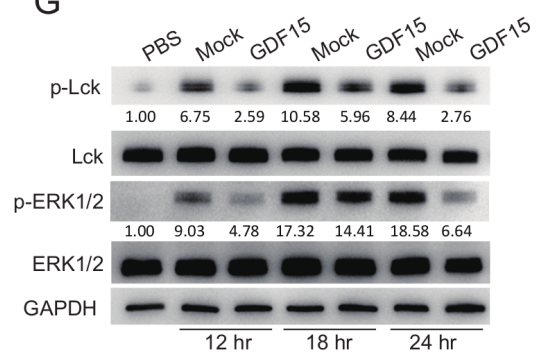

B

E
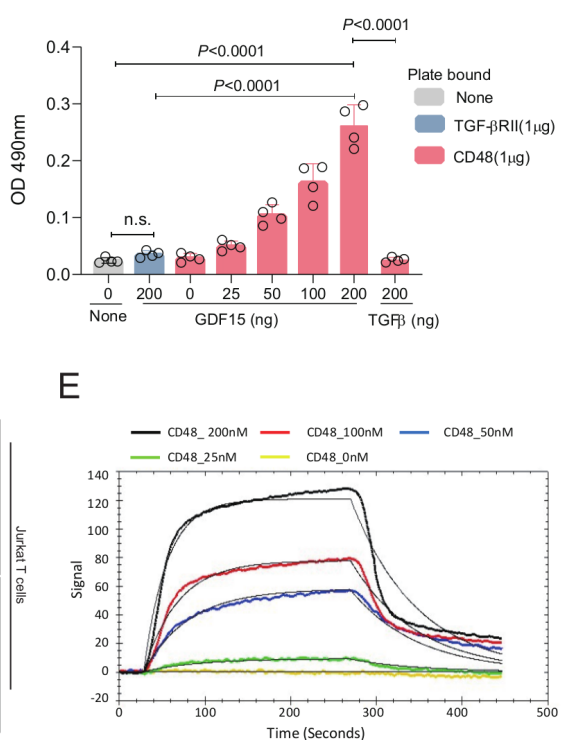

$\mathrm{H}$

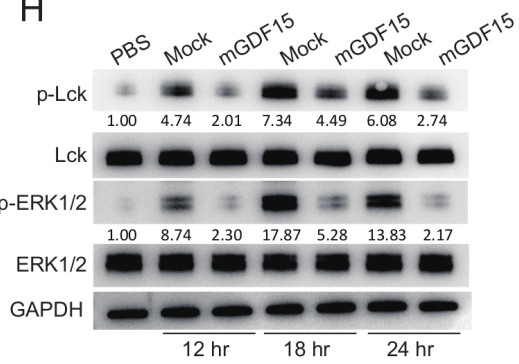

C

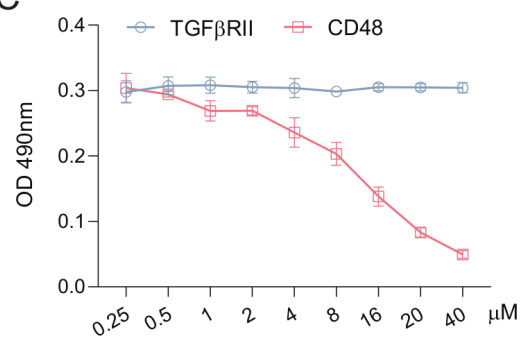

F

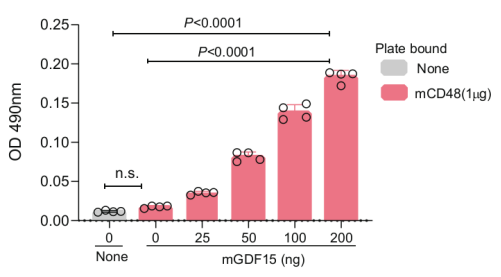

I

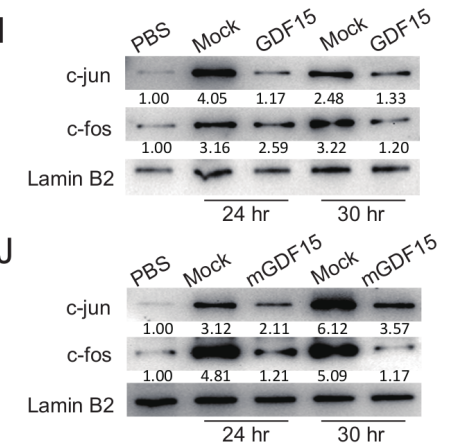

$\mathrm{K}$

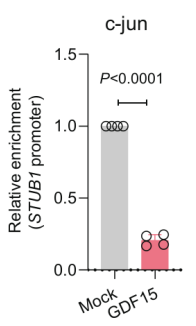

L

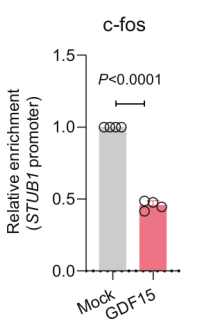

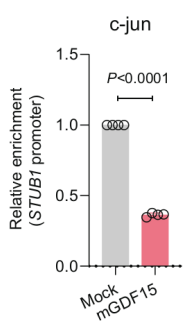

$\mathrm{M}$

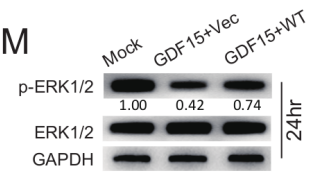

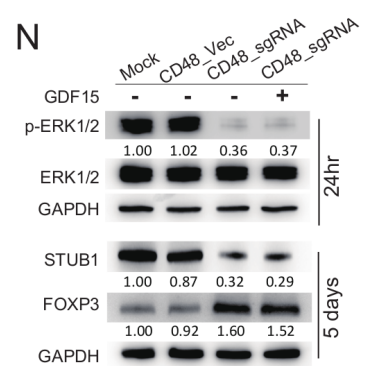

Figure 6 Growth differentiation factor 15 (GDF15) interacts with CD48 and downregulates STUB1 through inhibition of the ERK/AP-1 pathway. (A) Coimmunoprecipitation (ColP) analysis of the interaction between GDF15 and CD48. (B, C) ELISA confirmed GDF15 binds to CD48 in a dose-dependent manner and transforming growth factor $\beta$ (TGF- $\beta$ ) did not exhibit interaction with CD48 (B). The interaction of GDF15 to plate-bound CD48 could be blocked by the addition of soluble CD48 protein (C) $(n=4)$. (D) Representative immunofluorescence (IF) analysis of the interaction of GDF15 (red) with CD48 (green) on Jurkat T cells. (E) The interaction of GDF15 with purified CD48, as measured by surface plasmon resonance (SPR) spectroscopy. (F) ELISA confirmation of mouse GDF15 with mouse CD48 (n=4). (G-J) In human (G, I) and mouse (H, J) naïve $\mathrm{CD}^{+} \mathrm{T}$ cells, anti-CD3/anti-CD28 monoclonal antibodies (mAbs) and interleukin (IL)-2 stimulations with GDF15 (GDF15) or mouse GDF15 (mGDF15) led to a steady decline in the phosphorylation of Lck and ERK, and the decreasing of c-fos and $\mathrm{c}$-jun in nucleus. Expression levels of p-Lck and p-ERK were quantified and normalized to GAPDH expression. (K, L) Chromatin immunoprecipitation experiments showed that anti-CD3/anti-CD28 mAbs and IL-2 stimulations with GDF15 or mGDF15 significantly decreased the binding of c-jun and c-fos to the STUB1 promoter in human $(K)$ and mouse $(L)$ naïve $\mathrm{CD}^{+} \mathrm{T}$ cells ( $\mathrm{n}=4$ cell cultures). (M) The phosphorylation of ERK and the expressions of STUB1 and FOXP3 in unmanipulated Jurkat T cells after anti-CD28/anti-CD3 mAbs and IL-2 stimulations (Mock), CD48 gene (GDF15 +WT) or control vector (GDF15 +Vec) successfully transduced Jurkat T cells after anti-CD28/anti-CD3 mAbs and IL-2 stimulations with GDF15. (N) The phosphorylation of ERK and the expression of STUB1 and FOXP3 in unmanipulated (Mock), control knockout (CD48_Vec) and CD48 knockout Jurkat T cells after anti-CD28/anti-CD3 mAbs and IL-2 stimulations without (CD48_sgRNA) or with (CD48_sgRNA+GDF15) GDF15. Data are representative of two (E) or three (A-D, F-M) independent experiments. The intensities of target bands were quantified and normalized to GAPDH expression (G-J, M, N). P values were determined by a two-tailed unpaired t-test (B, F, K, L), n.s., not significant; $p>0.05$. 
knockout notably decreased the phosphorylation of ERK, downregulated the expression of STUB1 and increased the expression of FOXP3 compared with the control knockout, which mimicked the effects of GDF15 addition to unmanipulated cells. The addition of GDF15 did not affect the changes in ERK, STUB1 and FOXP3 caused by $\mathrm{CD} 48$ knockout (figure $6 \mathrm{~N}$ ). We further observed that CD48 knockout Jurkat T cells exhibited the phenotype of iTreg cells after activation. Similarly, GDF15 did not noticeably affect the generation of these iTreg cells (online supplemental figure 11J). Together, these data support that CD48 is the receptor of GDF15. The interaction of GDF15 with CD48 abolishes CD48-induced activation of the TCR downstream ERK/AP-1 pathway, and therefore downregulates STUB1. The downregulation of STUB1 thereafter causes FOXP3 accumulation.

\section{A GDF15 neutralizing mAb amplifies antitumor immunity}

At the end, we generate a GDF15-specific mAb (clone G15A) with specific neutralizing activity against both human and mouse GDF15 (online supplemental figure $12 \mathrm{~A}, \mathrm{~B})$. In vitro, G15A effectively inhibited the generation of iTreg cells induced by GDF15 (online supplemental figure 12C,D). We treated mice bearing Hepa1-6 cancer cells in the liver with G15A or isotype control antibody (Iso) (online supplemental figure 13A). Tumor volumes were significantly smaller in mice that received G15A than in mice that received Iso (figure 7A and B), and G15A markedly prolonged the survival of treated mice (figure 7C). A significantly lower frequency of Treg cells among $\mathrm{CD} 4^{+}$cells was found in the tumors and spleens of mice that received G15A (figure 7D). Ki67 expression was significantly upregulated in both $\mathrm{CD}^{+}$and $\mathrm{CD}^{+}$TILs of G15A-treated mice (figure 7E). In addition, G15A markedly increased the antitumor effects mediated by the anti-PD- $1 \mathrm{mAb}$ in mice (figure $7 \mathrm{~F}$ and $G$ ) and further prolonged the survival of treated mice (figure $7 \mathrm{H}$ ). One of the eight mice that received both G15A and anti-PD-1 antibodies exhibited complete tumor regression. Similarly, mice that received both G15A and the anti-PD-1 mAb had a lower frequency of Treg cells among $\mathrm{CD} 4^{+}$TILs (figure 7I) than mice that received only the anti-PD-1 mAb. Ki67 expression was significantly upregulated in both $\mathrm{CD} 4^{+}$and $\mathrm{CD}^{+}$(online supplemental figure 13B) TILs of mice that received the G15A and anti-PD-1 mAb combination. Finally, we transfused $\mathrm{OVA}_{257-264}$-activated OT-I T cells into Hepa1-6-OVAbearing mice. The combination of G15A and OT-I T cell transfusion demonstrated superior suppressive activity compared with OT-I T cell transfusion alone (figure $7 \mathrm{~J}$ ). Mice that received the combination treatment exhibited a decreased frequency of Treg cells among $\mathrm{CD} 4^{+}$TILs (figure 7K), and OT-I T cells recovered from tumors of mice synergistically treated with G15A and OT-I T cells exhibited enhanced proliferation (figure $7 \mathrm{~L}$ ). In all three models, we observed G15A significantly increased the food intake (online supplemental figure 14A) and weight of the mice (online supplemental figure 14B). Altogether, our observations support therapeutic blockade of GDF15 enhance antitumor immune response through Treg cells inhibition and achieve HCC clearance without obvious adverse events.

\section{DISCUSSION}

Nowadays, the immunomodulatory function of GDF15 is controversial. ${ }^{17}{ }^{23-26}$ Some studies of animal models of inflammation and autoimmune diseases ${ }^{24} 25940$ indicated that GDF15 has an overall beneficial effect on disease outcomes, which supports its immunosuppressive role. Limited reports on the mechanism attributed the immunosuppressive effects of GDF15 to the induction of inhibitory macrophages or neutrophils. ${ }^{23} 41$ Our results of orthotopic HCC models in $\mathrm{Rag} \mathrm{T}^{--}$mice and CyTOF analysis to the TILs indicate myeloid cell populations may not be the most important for the immunomodulatory function of GDF15 in HCC. For the first time, we show that severely elevated GDF15 expression in HCC cells and tissues negatively regulates antitumor immunity by promoting effects on Treg cells. We confirmed that GDF15 promotes the generation of iTreg cells and the suppressive function of nTreg cells by interacting with CD48, which drives GDF15-mediated post-transcriptional regulation of FOXP3. As a member of the TGF- $\beta$ family, GDF15 was previously considered to exert functions through the TGF- $\beta$ receptor. Limited studies on the mechanism of GDF15 in macrophages and neutrophils also suggest that GDF15 functions through the downstream canonical or non-canonical pathways of TGF- $\beta .^{23}{ }^{41}$ Unfortunately, several recent studies reported that GDF15 does not bind to TGF- $\beta$ receptors 1 and 2 or to any of the 139 other molecules related to the TGF- $\beta$ receptor superfamily and that GDF15 does not activate any pathways downstream of the TGF- $\beta$ receptor. ${ }^{19-22}$ GFRAL is currently the only recognized receptor of GDF15 and is responsible for the central modulation of appetite by GDF15. Intriguingly, GFRAL is exclusively expressed in hindbrain neurons and is not present in any peripheral tissues, including lymph nodes, thymus and bone marrow. ${ }^{20}{ }^{21}$ These findings make elucidating the molecular mechanism by which GDF15 regulates the immune system challenging. CD48 is a glycosyl-phosphatidyl-inositol (GPI)-anchored protein expressed only on leukocytes. On T cells, after interaction with ligands CD2 or CD244 in cis or trans, CD48 associates with the TCR complex, activates the ERK pathway and then contributes to the activation of T cells. ${ }^{36}{ }^{37} \mathrm{In}$ the present study, we identified CD48 as a novel receptor of GDF15. The SPR results revealed that the GDF15-CD48 interaction had a $\mathrm{Kd}$ of $\sim 0.1 \mu \mathrm{M}$, which is much higher than that between CD48 and CD2 (Kd $\sim 100 \mu \mathrm{M})$ and between $\mathrm{CD} 48$ and $\mathrm{CD} 244(\mathrm{Kd} \sim 8 \mu \mathrm{M})$. Therefore, as a secreted molecule, GDF15 competitively inhibits the binding of CD48 to its ligands CD2 and CD244, thereby inhibiting the downstream pathway of CD48. CD48 is the first discovered receptor of GDF15 in the immune system and is exclusively expressed on all leukocytes. It 
A

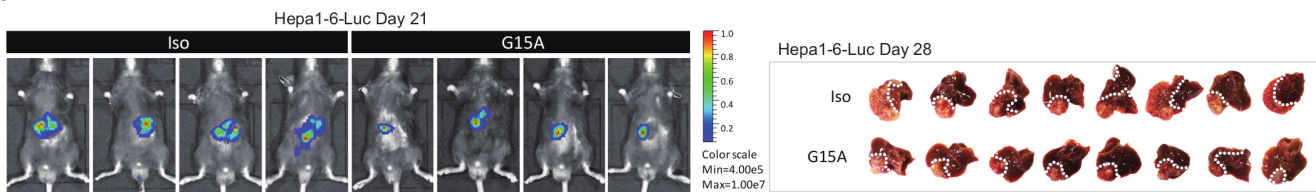

B

C
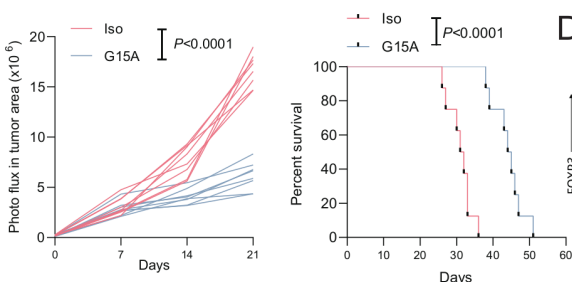

E
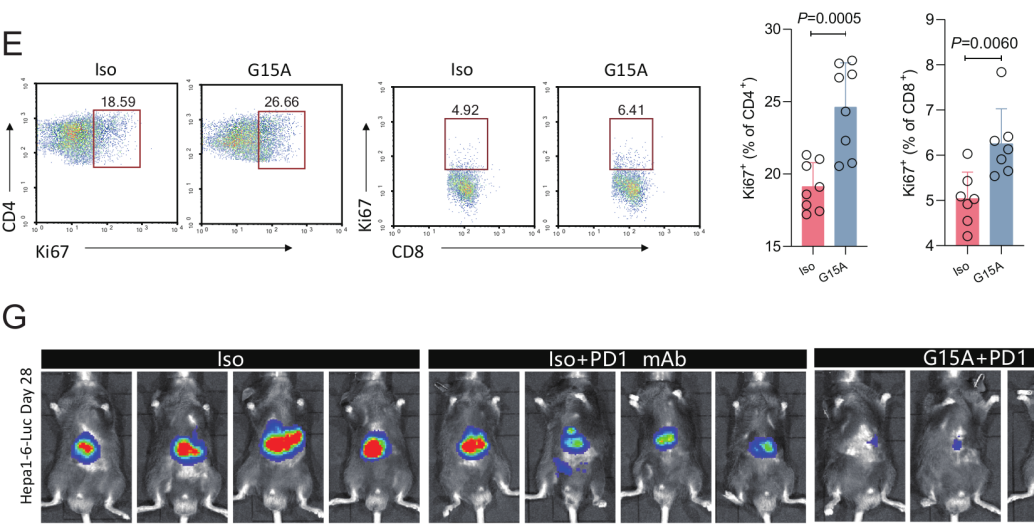

F
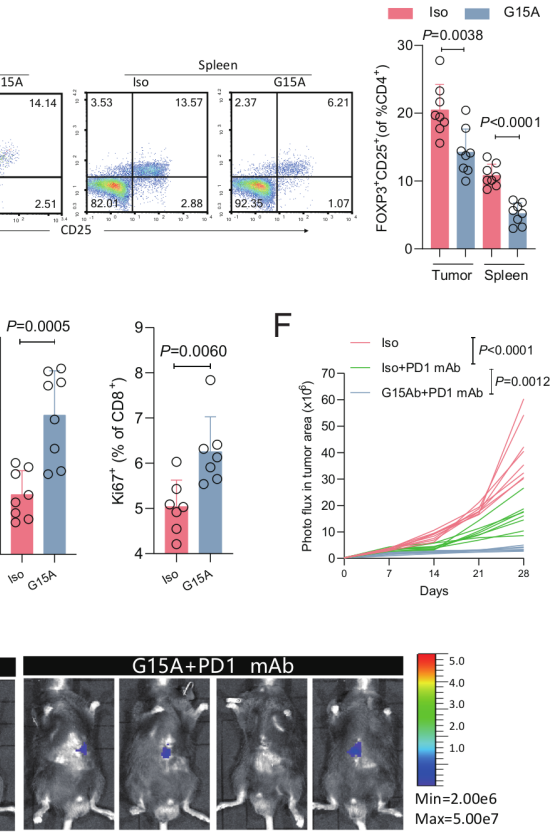

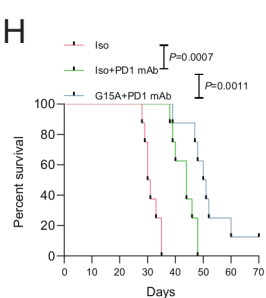

K

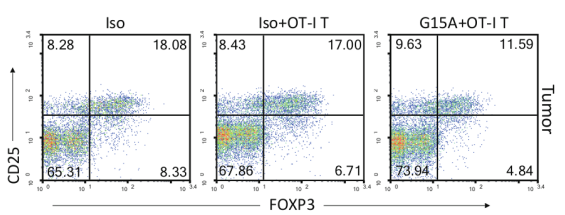

।
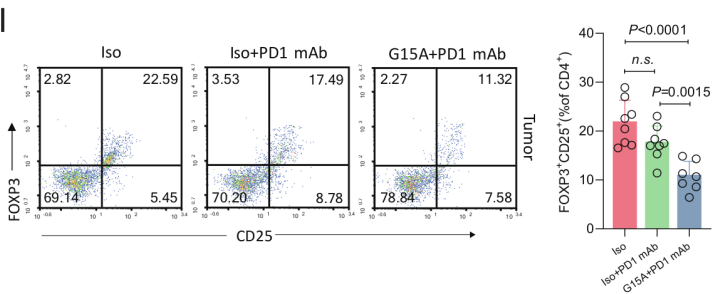

J

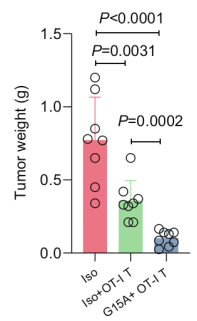

L
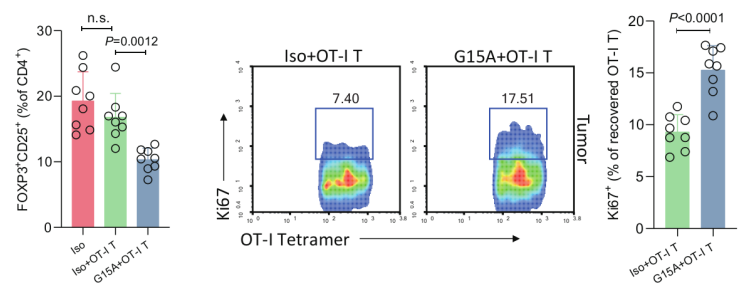

Figure 7 A growth differentiation factor 15 (GDF15) neutralizing monoclonal antibody (mAb) (G15A) amplifies antitumor immunity in mice. (A-E) Mice carrying Hepa1-6-Luc cells in livers were treated with G15A or control antibody (Iso) (A, $n=8$ mice per group). Tumor growth was monitored by values of bioluminescence $\left(\mathrm{p} / \mathrm{s} / \mathrm{cm}^{2} / \mathrm{sr}\right)(B)$. Representative bioluminescence imaging of four mice in each group on day 21 and liver images of all 16 mice acquired on day 28 after euthanasia are shown (A, the tumor is identified by the dotted line). Survival was evaluated using another two groups of mice (C, $n=8$ mice per group). The frequencies of regulatory $\mathrm{T}$ (Treg) cells among CD4 $4^{+}$tumor-infiltrating lymphocytes (TILs) and splenic CD4 ${ }^{+} \mathrm{T}$ cells (D), and Ki67 expression in $\mathrm{CD}^{+}$and $\mathrm{CD} 8^{+}$TILs (E) on day 28 after euthanasia are shown. (F-l) Mice carrying Hepa1-6-Luc cell in livers were treated with Iso, Iso plus antimouse programmed cell death protein 1 (PD-1) antibody (PD-1 mAb) or G15A plus PD-1 $\mathrm{mAb}\left(\mathrm{n}=8\right.$ mice per group). Tumor growth was monitored by bioluminescence $\left(\mathrm{p} / \mathrm{s} / \mathrm{cm}^{2} / \mathrm{sr}\right)(\mathrm{F})$. Representative bioluminescence imaging of four mice in each group at day 21 are shown $(G)$. Survival was evaluated in another three groups of mice $(H, n=8$ mice per group). The frequencies of Treg cells among CD4 ${ }^{+}$TILs cells on 28 days after euthanasia are shown (I). (J-L) Mice carrying Hepa1-6-OVA cells in livers were treated with Iso, Iso plus OT-I T cells or G15A plus OT-I T cells. Tumor weights at day 21 after mice were euthanized $(\mathrm{J})$, the frequency of Treg cells among CD4 $4^{+}$TILs $(\mathrm{K})$ and Ki67 expression in OT-I T cells in tumors on day 21 are shown (L). Data are representative of three independent experiments. $P$ values were determined by two-tailed Mann-Whitney $U$ test $(B, G)$, log rank test $(C, H)$ or two-tailed unpaired t-test (D, E, I-K), Iso, isotype control; n.s., not significant; $\mathrm{p}>0.05$. 
is possible that the previously reported effects of GDF15 on different immune cells may also be achieved through CD48. How GDF15 functions through CD48 on different immune cells and whether some rapid and transient antiinflammatory functions of GDF15 reported in myocardial infarction $^{24}$ are mediated by CD48 are worthy of further research. In addition, it may be more reasonable to call CD48 a binding partner of GDF15 rather than receptor because CD48 cannot transmit signals alone.

Regardless of the mechanism of concern, our findings and some studies, including epidemiological studies of different cancers, support the notion of GDF15 in promoting tumor development. ${ }^{22}{ }^{41-43}$ However, some studies using Gdf15 genetically modified mice to study early tumorigenesis have reported conflicting conclusions. For instance, in a DEN-induced HCG model in Gdf15 - mice, Zimmer et al indicated that GDF15 has no role in hepatocarcinogenesis. ${ }^{44}$ By hybridizing Gdf15 ${ }^{\circ-}$ mice and intestinal neoplasia-prone mice bearing the mutant adenomatous polyposis coli $(A p c)$ gene, they showed that GDF15 has no role in intestinal neoplasia formation again. ${ }^{45}$ By the same hybridization method, Baek et al believe that GDF15 has an inhibitory effect on intestinal neoplasia formation. ${ }^{46}$ Based on the standard DEN induction method, we think that the procedure used by Zimmer et alneeds to be optimized, and the nodules they observed may not be pathological HCC. ${ }^{47}$ On the other hand, from our point, GDF15 promotes tumor development through its immunosuppressive function, which depends on the formation of an adaptive antitumor immune response in vivo. Thus, we think transgenic cancer-prone mice are not suitable for GDF15 research, as germline manipulations of oncogenes can trigger immune tolerance to the relevant antigen during embryonic development, which eventually causes no antitumor immune response in adult mice. In our work, mutated oncogenes were transfected into the livers of adult $G d f 15^{--}$mice via hydrodynamic injection, which can effectively elicit an appropriate antitumor immune response. Therefore, we think our results that GDF15 promotes hepatocarcinogenesis are more convincing; after all, most tumorigenesis is caused by acquired mutations in oncogenes. Besides, some studies in other tumor types show that GDF15 achieves its tumor promotion effect through directly facilitates the growth of cancer cells. ${ }^{48} 49$ However, according to our real-time cell impedance assay and orthotopic HCC model in Rag $1^{-}$ - mice, we believe that GDF15 promotes carcinogenesis and the development of established tumors via immunosuppression, at least in HCC.

Currently, HCC is the third leading cause of cancerrelated death globally. Given the common failures of chemotherapies and targeted therapies in the field of HCC treatment, promising breakthroughs are eagerly needed. ${ }^{50}$ Our results indicate that targeting GDF15 with an antibody can convert an immunosuppressive TME to an inflammatory TME and achieve HCC clearance. Since the physiological level of GDF15 is low and $G d f 15^{-}$mice exhibit no apparent disease phenotypes, we predict that
GDF15 inhibition will not disrupt normal physiological functions or induce deleterious autoimmune responses, unlike immune checkpoint suppression therapy. Moreover, we observed that GDF15 blockade therapy ameliorates tumor-associated anorexia, which is expected, as GDF15 upregulation in disease states can suppress appetite through a central mechanism. ${ }^{19-22}$ Collectively, GDF15 is a tumor-derived immunosuppressive molecule and a remarkable target for cancer immunotherapy of HCC. Of course, there must be other molecules related to the immunosuppression of HCC, which are worthy of further study.

In summary, our study demonstrates that GDF15, as an HCC-derived immunosuppressive molecule, promotes the generation of iTreg cells and the suppressive function of nTreg cells by interacting with CD48, which mediates GDF15-mediated post-transcriptional regulation of FOXP3. CD48 is the first discovered receptor of GDF15 in the immune system. Considering its unique features, the GDF15-neutralizing mAb may augment the therapeutic tools available for HCC, which has a poor response to current immunotherapies.

\section{Author affiliations}

${ }^{1}$ State Key Laboratory of Cancer Biology, Fourth Military Medical University, Xi'an, Shaanxi, China

${ }^{2}$ Department of Hepatobiliary Surgery, Xijing Hospital, Fourth Military Medical University, Xi'an, Shaanxi, China

${ }^{3}$ Department of Immunology, Fourth Military Medical University, Xi'an, Shaanxi, China

${ }^{4}$ Department of Health Statistics, Fourth Military Medical University, Xi'an, Shaanxi, China

${ }^{5}$ Department of oncology, Tangdu Hospital, Fourth Military Medical University, Xi'an, Shaanxi, China

Acknowledgements We thank Dr Se-Jin Lee of Johns Hopkins University School of Medicine for technical support in Gdf15 knockout mice construction; Dr Xin Chen of University of California, San Francisco for giving myr-AKT1, N-RasV12 and sleeping beauty transposase plasmids; Ying Tian of Department of Immunology, Fourth Military Medical University for technical assistance in antibody production and other members in the laboratories of Fourth Military Medical University for helpful discussions.

Contributors $\mathrm{ML}, \mathrm{YZ}$ and $\mathrm{CZ}$ designed the study, interpreted data and wrote the manuscript. ZW conducted the orthotopic tumor models and in vitro experiments. LH conducted the western blot analysis and immunoprecipitation experiments. WL conducted the in vitro experiments and cell transfer assays. RZ, BJ, LS, ZT, WZ (Wei Zhang) and HS helped with the design of experiments and coordinated the study. LH, QH, KZ, WZ (Wangqian Zhang) and SW contributed key reagents. DW and KD led liver cancer clinical team/core that provided samples for analysis. CX, JZ, JG and YG conducted immunohistochemistry, pathological analysis, assisted mouse model studies and immune phenotyping.

Funding This research was supported by the National Natural Science Foundation of China $(81673020,81703407,81672800,81672864,81702590$ and 81802632) and Shaanxi Natural Science Foundation (2017ZDCXL-SF-01-03).

Competing interests None declared.

Patient consent for publication Not required.

Ethics approval All samples were collected from patients with informed consent, and all related procedures were performed with the approval of the internal review and ethics boards of Xijing Hospital (XJYYLL-2014465), Fourth Military Medical University, Xi'an, China.

Provenance and peer review Not commissioned; externally peer reviewed.

Data availability statement Data are available in a public, open access repository. All data relevant to the study are included in the article or uploaded as 
supplementary information. Availability of data and material: the RNA-seq data were deposited in the NCBI Sequence Read Archive (https://www.ncbi.nlm.nih.gov/sra/) under SRA accession number PRJNA627605.

Supplemental material This content has been supplied by the author(s). It has not been vetted by BMJ Publishing Group Limited (BMJ) and may not have been peer-reviewed. Any opinions or recommendations discussed are solely those of the author(s) and are not endorsed by BMJ. BMJ disclaims all liability and responsibility arising from any reliance placed on the content. Where the content includes any translated material, BMJ does not warrant the accuracy and reliability of the translations (including but not limited to local regulations, clinical guidelines, terminology, drug names and drug dosages), and is not responsible for any error and/or omissions arising from translation and adaptation or otherwise.

Open access This is an open access article distributed in accordance with the Creative Commons Attribution Non Commercial (CC BY-NC 4.0) license, which permits others to distribute, remix, adapt, build upon this work non-commercially, and license their derivative works on different terms, provided the original work is properly cited, appropriate credit is given, any changes made indicated, and the use is non-commercial. See http://creativecommons.org/licenses/by-nc/4.0/.

\section{ORCID iD}

Meng Li http://orcid.org/0000-0002-8000-3003

\section{REFERENCES}

1 Hegde PS, Chen DS. Top 10 challenges in cancer immunotherapy. Immunity 2020;52:17-35.

2 El-Khoueiry AB, Sangro B, Yau T, et al. Nivolumab in patients with advanced hepatocellular carcinoma (CheckMate 040): an open-label, non-comparative, phase 1/2 dose escalation and expansion trial. Lancet 2017;389:2492-502.

3 Robert C, Schachter J, Long GV, et al. Pembrolizumab versus ipilimumab in advanced melanoma. N Engl J Med 2015;372:2521-32.

4 Motzer RJ, Escudier B, McDermott DF, et al. Nivolumab versus everolimus in advanced renal-cell carcinoma. $N$ Engl J Med 2015;373:1803-13.

5 Garon EB, Hellmann MD, Rizvi NA, et al. Five-Year overall survival for patients with advanced Non-Small-Cell lung cancer treated with pembrolizumab: results from the phase I KEYNOTE-001 study. J Clin Oncol 2019;37:2518-27.

6 Adeegbe DO, Nishikawa $\mathrm{H}$. Natural and induced T regulatory cells in cancer. Front Immunol 2013;4:190.

7 De Simone M, Arrigoni A, Rossetti G, et al. Transcriptional landscape of human tissue lymphocytes unveils uniqueness of tumor-infiltrating T regulatory cells. Immunity 2016;45:1135-47.

8 Ormandy LA, Hillemann T, Wedemeyer $\mathrm{H}$, et al. Increased populations of regulatory T cells in peripheral blood of patients with hepatocellular carcinoma. Cancer Res 2005;65:2457-64.

9 Zheng C, Zheng L, Yoo J-K, et al. Landscape of infiltrating T cells in liver cancer revealed by single-cell sequencing. Cell 2017;169:e1316:1342-56.

10 Makarova-Rusher OV, Medina-Echeverz J, Duffy AG, et al. The yin and yang of evasion and immune activation in HCC. $J$ Hepatol 2015;62:1420-9.

11 Saito T, Nishikawa H, Wada H, et al. Two FOXP3(+)CD4(+) T cell subpopulations distinctly control the prognosis of colorectal cancers. Nat Med 2016;22:679-84.

12 Zhang Y, Lazarus J, Steele NG, et al. Regulatory T-cell depletion alters the tumor microenvironment and accelerates pancreatic carcinogenesis. Cancer Discov 2020;10:422-39.

13 Bos PD, Plitas G, Rudra D, et al. Transient regulatory $T$ cell ablation deters oncogene-driven breast cancer and enhances radiotherapy. $J$ Exp Med 2013;210:2435-66.

14 Liu C, Chikina M, Deshpande R, et al. Treg Cells Promote the SREBP1-Dependent Metabolic Fitness of Tumor-Promoting Macrophages via Repression of $\mathrm{CD}^{+} \mathrm{T}$ Cell-Derived Interferon- $\gamma$. Immunity 2019;51:381-97.

15 Nyström SN, Bourges D, Garry S, et al. Transient Treg-cell depletion in adult mice results in persistent self-reactive CD4(+) T-cell responses. Eur J Immunol 2014;44:3621-31.

16 Uhlen M, Zhang C, Lee S, et al. A pathology atlas of the human cancer transcriptome. Science2017;357:eaan2507.

17 Luan HH, Wang A, Hilliard BK, et al. GDF15 is an inflammationinduced central mediator of tissue tolerance. Cell 2019;178:1231-44.
18 Zhang S, Dai D, Wang X, et al. Growth differentiation factor-15 predicts the prognoses of patients with acute coronary syndrome: a meta-analysis. BMC Cardiovasc Disord 2016;16:82

19 Yang L, Chang C-C, Sun Z, et al. GFRAL is the receptor for GDF15 and is required for the anti-obesity effects of the ligand. Nat Med 2017;23:1158-66.

20 Mullican SE, Lin-Schmidt X, Chin C-N, et al. GFRAL is the receptor for GDF15 and the ligand promotes weight loss in mice and nonhuman primates. Nat Med 2017;23:1150-7

21 Emmerson PJ, Wang F, Du Y, et al. The metabolic effects of GDF15 are mediated by the orphan receptor GFRAL. Nat Med 2017;23:1215-9.

22 Tsai VWW, Husaini Y, Sainsbury A, et al. The MIC-1/GDF15-GFRAL pathway in energy homeostasis: implications for obesity, cachexia, and other associated diseases. Cell Metab 2018;28:353-68.

23 Artz A, Butz S, Vestweber D. GDF-15 inhibits integrin activation and mouse neutrophil recruitment through the ALK-5/TGF- $\beta$ RII heterodimer. Blood 2016;128:529-41.

24 Kempf T, Zarbock A, Widera C, et al. GDF-15 is an inhibitor of leukocyte integrin activation required for survival after myocardial infarction in mice. Nat Med 2011;17:581-8.

25 Abulizi P, Loganathan N, Zhao D, et al. Growth differentiation factor-15 deficiency augments inflammatory response and exacerbates septic heart and renal injury induced by lipopolysaccharide. Sci Rep 2017;7:1037.

26 Harb H, Stephen-Victor E, Crestani E, et al. A regulatory T cell Notch4-GDF15 axis licenses tissue inflammation in asthma. Nat Immunol 2020;21:1359-70.

27 Ansa-Addo EA, Zhang Y, Yang Y, et al. Membrane-organizing protein moesin controls Treg differentiation and antitumor immunity via TGF- $\beta$ signaling. J Clin Invest 2017;127:1321-37.

28 Chen W, Jin W, Hardegen N, et al. Conversion of Peripheral CD4 ${ }^{+} \mathrm{CD} 25^{-}$Naive T Cells to $\mathrm{CD} 4^{+} \mathrm{CD} 25^{+}$Regulatory T Cells by TGF- $\beta$ Induction of Transcription Factor Foxp3. J Exp Med 2003;198:1875-86.

29 Chen Z, Barbi J, Bu S, et al. The ubiquitin ligase STUB1 negatively modulates regulatory $T$ cell suppressive activity by promoting degradation of the transcription factor FOXP3. Immunity 2013;39:272-85

30 Ho C, Wang C, Mattu S, et al. AKT (v-akt murine thymoma viral oncogene homolog 1) and N-Ras (neuroblastoma ras viral oncogene homolog) coactivation in the mouse liver promotes rapid carcinogenesis by way of mTOR (mammalian target of rapamycin complex 1), FOXM1 (forkhead box M1)/SKP2. Hepatology 2012;55:833-45.

31 R.Walker M, Kasprowicz DJ, Gersuk VH, et al. Induction of FoxP3 and acquisition of $\mathrm{T}$ regulatory activity by stimulated human CD4 ${ }^{+}$CD25- T cells. J. Clin. Invest. 2003;112:1437-43

32 Gottschalk RA, Corse E, Allison JP. TCR ligand density and affinity determine peripheral induction of Foxp3 in vivo. $J$ Exp Med 2010;207:1701-11

33 Allan SE, Crome SQ, Crellin NK, et al. Activation-Induced FOXP3 in human $T$ effector cells does not suppress proliferation or cytokine production. Int Immunol 2007;19:345-54.

34 Godoy GJ, Olivera C, Paira DA, et al. T regulatory cells from nonobese diabetic mice show low responsiveness to IL-2 stimulation and exhibit differential expression of Anergy-Related and ubiquitination factors. Front Immunol 2019;10:2665.

$35 \mathrm{Li} \mathrm{MO}$, Rudensky AY. T cell receptor signalling in the control of regulatory $\mathrm{T}$ cell differentiation and function. Nat Rev Immunol 2016;16:220-33.

36 Moran M, Miceli MC. Engagement of GPI-linked CD48 contributes to TCR signals and cytoskeletal reorganization: a role for lipid rafts in T cell activation. Immunity 1998;9:787-96.

37 McArdel SL, Terhorst C, Sharpe AH. Roles of CD48 in regulating immunity and tolerance. Clin Immunol 2016;164:10-20.

38 Wang J, Zhang W, Wang S, et al. Swine-Derived probiotic Lactobacillus plantarum modulates porcine intestinal endogenous host defense peptide synthesis through TLR2/MAPK/AP-1 signaling pathway. Front Immunol 2019;10:2691.

39 Johnen H, Kuffner T, Brown DA, et al. Increased expression of the TGF-b superfamily cytokine MIC-1/GDF15 protects ApoE-/mice from the development of atherosclerosis. Cardiovasc Pathol 2012;21:499-505.

40 Tanrıkulu O, Sarıyıldız MA, Batmaz İbrahim, et al. Serum GDF15 level in rheumatoid arthritis: relationship with disease activity and subclinical atherosclerosis. Acta Reumatol Port 2017;42:66-72.

41 Ratnam NM, Peterson JM, Talbert EE, et al. NF- $\kappa B$ regulates GDF-15 to suppress macrophage surveillance during early tumor development. J Clin Invest 2017;127:3796-809. 
42 Liu Y-N, Wang X-B, Wang T, et al. Macrophage inhibitory cytokine-1 as a novel diagnostic and prognostic biomarker in stage I and II nonsmall cell lung cancer. Chin Med $J$ 2016;129:2026-32.

43 Lu L, Ma G-Q, Liu X-D, et al. Correlation between GDF15, MMP7 and gastric cancer and its prognosis. Eur Rev Med Pharmacol Sci 2017;21:535-41.

44 Zimmers TA, Jin X, Gutierrez JC, et al. Effect of in vivo loss of GDF-15 on hepatocellular carcinogenesis. J Cancer Res Clin Oncol 2008;134:753-9.

45 Zimmers TA, Gutierrez JC, Koniaris LG. Loss of GDF-15 abolishes sulindac chemoprevention in the ApcMin/+ mouse model of intestinal cancer. J Cancer Res Clin Oncol 2010;136:571-6.

46 Baek SJ, Okazaki R, Lee S-H, et al. Nonsteroidal antiinflammatory drug-activated gene- 1 over expression in transgenic mice suppresses intestinal neoplasia. Gastroenterology 2006;131:1553-60.

47 Tolba R, Kraus T, Liedtke C, et al. Diethylnitrosamine (DEN)-induced carcinogenic liver injury in mice. Lab Anim 2015;49:59-69.

48 Zhao Z, Zhang J, Yin L, et al. Upregulated GDF-15 expression facilitates pancreatic ductal adenocarcinoma progression through orphan receptor GFRAL. Aging 2020;12:22564-81.

49 Meier JC, Haendler B, Seidel H, et al. Knockdown of platinuminduced growth differentiation factor 15 abrogates p27-mediated tumor growth delay in the chemoresistant ovarian cancer model A2780cis. Cancer Med 2015;4:253-67.

50 Siu EH-L, Chan AW-H, Chong CC-N, et al. Treatment of advanced hepatocellular carcinoma: immunotherapy from checkpoint blockade to potential of cellular treatment. Trans/ Gastroenterol Hepatol 2018;3:89. 